\title{
Multi-item quick response system with budget constraint
}

\author{
Doğan A. Serel* \\ Faculty of Business Administration, Bilkent University, 06800 Bilkent, Ankara, Turkey
}

\section{A R T I C L E I N F O}

\section{Article history:}

Received 20 December 2010

Accepted 20 January 2012

Available online 10 February 2012

\section{Keywords:}

Inventory

Multi-product newsvendor problem

Budget constraint

Forecast update

Bayesian estimate

\begin{abstract}
A B S T R A C T
Quick response mechanisms based on effective use of up-to-date demand information help retailers to reduce their inventory management costs. We formulate a single-period inventory model for multiple products with dependent (multivariate normal) demand distributions and a given overall procurement budget. After placing orders based on an initial demand forecast, new market information is gathered and demand forecast is updated. Using this more accurate second forecast, the retailer decides the total stocking level for the selling season. The second order is based on an improved demand forecast, but it also involves a higher unit supply cost. To determine the optimal ordering policy, we use a computational procedure that entails solving capacitated multi-item newsboy problems embedded within a dynamic programming model. Various numerical examples illustrate the effects of demand variability and financial constraint on the optimal policy. It is found that existence of a budget constraint may lead to an increase in the initial order size. It is also observed that as the budget available decreases, the products with more predictable demand make up a larger share of the procurement expenditure.
\end{abstract}

(c) 2012 Elsevier B.V. All rights reserved.

\section{Introduction}

A common problem faced by many retailers is the determination of the optimal stocking quantity prior to a single selling season in which customer demand for a product is uncertain. Given a demand forecast and cost estimates for leftovers and unsatisfied demand, the optimal order quantity can be decided using the well-known newsvendor model (see e.g. Silver et al., 1998). Since 1990s quick response systems that enable the retailers to place two different orders with their suppliers before the selling season have been popular in supply chains of seasonal products such as fashion apparel, footwear, and toys (Fisher and Raman, 1996; Iyer and Bergen, 1997; Perry et al., 1999). Reducing lead times throughout all the production-supply chain activities is a primary focus of quick response manufacturing strategy (Fernandes and Carmo-Silva, 2006). Instead of placing a traditional single order, in a quick response setting, a retailer can implement the following strategy: an initial order is placed long before the selling season when the supplier is willing to commit to a low supply price, and a second order is placed at a time closer to the selling season when the retailer has a better assessment of the potential demand. It is likely that the supply price will be higher for the second order. For example, a cheaper off-shore supplier becomes an eligible source when the lead time is long

\footnotetext{
* Tel.: +90 312290 2415; fax: +90312266 4958 .

E-mail address: serel@bilkent.edu.tr
}

whereas a short lead time may necessitate using a high-cost domestic supplier. When a supplier is allowed a short delivery time, it may ask a higher supply price because it is more difficult to acquire and utilize cost-effective means of production and transportation within a short time frame. When the supplier has more time to complete production, it is possible to procure raw materials at a lower cost and develop more efficient production schedules. These cost savings realized by the supplier may translate into a lower purchase price for the retailer.

Although a short lead time may necessitate an increase in the purchase cost for the retailer, nevertheless it offers a possibility to decrease the demand forecast error (Bitran et al., 1986). A more accurate demand forecast enables the retailer to choose a more appropriate stocking level, and hence decrease its expected inventory costs. Thus the retailer can try to optimally balance the tradeoff between demand forecast error and supply cost by placing two separate orders at two different times before the season. The first order takes advantage of the low supply cost while the second order utilizes an improved demand forecast.

Fisher and Raman (1996) describe in detail how a fashion skiwear designer and manufacturer, Sport Obermeyer, has applied the quick response approach in developing production schedules for a group of products with varying demand forecast characteristics. Their model has two production periods as the setup costs and other economies of scale make it undesirable to manufacture a product more than twice in a selling season.

In this paper we explore the problem of a newsvendor who places two different orders for multiple products before the 
season given that demand forecast is updated based on market signals received between the times of two orders. The demands for products are assumed to be distributed as multivariate normal with an unknown mean. The market signals received after the first order are used to update the estimate of the mean of the multivariate demand distribution. For each product, the size of the second order is contingent on the market information collected. More encouraging demand signals increase the size of the second order.

In practice, following the preliminary demand forecast, new market information for the products can be obtained via various sources such as marketing research, trade shows, and early order commitments (Donohue, 2000). Actual sales observations for similar products can be used to revise the demand forecast of a product (Iyer and Bergen, 1997). Other sources of data include mock stores, fashion shows, focus groups and consulting experts (Caro and Martinez-de-Albeniz, 2010).

The differentiating feature of our work is that we consider the existence of a budget constraint for purchases, and also we extend a particular single-product model studied in the quick response literature to a multi-product setting. By incorporating the features of budget constraint and multiple products into the basic model, we can study how the optimal ordering policy in a quick response system changes with changes in the availability of financial resources, and parameters of the multivariate demand distribution. The retailer's decision problem is to maximize its total expected profit, and it is solved using a two-stage stochastic dynamic programming model. For the budget-constrained singleproduct problem, we show several properties of the optimal ordering policy. For the multi-product problem, these properties are verified in a numerical study.

The budget-constrained, multi-product, single-period inventory problem under a single demand forecast with known distribution parameters has been explored by many researchers. By making some distribution parameters unknown, and allowing an option of a second order prior to the selling season based on a revised demand forecast, we integrate this classical problem with the literature on quick response retail operations. When the budget of the newsvendor is limited, the ordering decision will be influenced by the relative profitability of different products as well as the improvement potential in demand forecasts. If the product demands are correlated with each other, the order quantity for a particular product should be decided by taking into account the demand information associated with other products.

An interesting result from our study is that limiting the funds earmarked for purchasing a product may lead to an increase in the quantity ordered prior to collecting the market signal. The reason is that when it is almost certain that all money will be spent eventually, it is desirable to reduce the average supply cost per unit in order to start the season with as much stock as possible. Conversely, increasing the amount of available funds causes a drop in the initial order quantity as the risk of facing a financial constraint at the time of the second order decreases.

In our numerical study we observe that the optimal budget allocation among the products at the first stage depends on the degree of demand uncertainty as well as the amount of funds available. While products with more predictable demand are favored under limited budget conditions, the removal of the financial constraint results in a significant increase in the purchase quantity of products with more volatile demand.

The computational study suggests that collecting new market information to improve the demand forecast yields a significant benefit only when the procurement budget is sufficiently large. A restrictive budget leaves the retailer with limited funds that can be used at the second stage, which places an upper bound on the cost savings from demand forecast update. It is also observed that a higher purchase cost at the second stage induces the retailer to increase its initial order so that a possibly large expenditure at the second stage can be avoided.

The remainder of the paper is structured as follows. We first review the related literature on quick response and the multiitem newsboy problem in Section 2, and describe how parameter estimation is carried out in our model in Section 3. We study two special cases of the procurement optimization problem separately in Sections 4 and 5. In Section 4, we discuss the solution of the multi-product problem when the budget constraint is not binding. The single-product problem subject to a budget constraint is explored in Section 5. In Section 6, we look into the most general problem, that is, the multi-product problem with a constraining budget. For this problem, we propose a computational optimization procedure that entails solving a series of multi-item newsvendor problems. We present some numerical examples in Section 7, and offer suggestions for future research in Section 8.

\section{Literature review}

The issues of demand information sharing in supply chain management and the tradeoff between the cost and responsiveness of the order fulfillment process due to the presence of dual supply modes have been investigated by many researchers (e.g., Zhu et al., 2011; Bhatnagar et al., 2011; Klosterhalfen et al., 2011). Our paper is mainly related to two different research streams. The first line of research consists of papers with analytical models of quick response systems. Since the literature is extensive, we review here a few representative works only. For a more detailed review, see e.g., Choi and Sethi (2010), and Cheng and Choi (2010). The papers in this area contain multi-period production or ordering problems in which demand forecast is revised in each period. Murray and Silver (1966) formulate a Bayesian updating model in which a retailer has multiple ordering opportunities for a product during the selling season. The demand for the product is an unknown proportion of the known total demand for a group of products. As the season progresses and sales figures are observed, the retailer updates its estimate of the unknown parameter and decides its order size accordingly at each ordering instant. Bitran et al. (1986) develop a multi-item, multi-period production model in which demand for each item is concentrated in the last period; the forecast for each item is revised before determining the production quantities in each period. Matsuo (1990) removes some restrictions in Bitran et al. (1986), and studies a two-stage stochastic sequencing problem.

Our work is more closely related to the papers that allow two production (or ordering) opportunities. The papers in this group can be further classified based on whether the second-stage production decision is made prior to or during the selling season. Fisher and Raman (1996) develop a multi-product model in which a manufacturer divides the production time available into two periods. In the first period a production run is made for each product without receiving any customer order. After receiving some customer orders, another batch is produced in the second period. Fisher and Raman (1996) constrain the batch size of each product to a specific range bounded by a set of lower and upper limits. Raman and Kim (2002) explore a similar problem with more than two periods. Iyer and Bergen (1997) study how the establishment of a quick response link influences the profits of the retailer and the manufacturer. Donohue (2000) looks into how buy-back contracts can achieve channel coordination in a manufacturer-retailer channel when the retailer updates demand forecast before placing its second order. Choi and Chow (2008) study the effect of various contracting schemes on the mean and 
variance of profit distributions in a quick response supply chain. Li et al. (2009) investigate a problem where the time of the second order is also a decision variable. Caro and Martinez-de-Albeniz (2010) formulate a two-period, two-retailer inventory competition model to study the competitive advantage gained via demand information updates with respect to the traditional slow-response retail operations.

Although most two-stage ordering models assume that the unit production (or purchase) cost is higher at the second stage, Gurnani and Tang (1999) allow cost uncertainty and possibly lower cost at the second stage. Choi et al. (2003) study a similar problem in which the demand is assumed to follow a normal distribution with an unknown mean, and a Bayesian approach is used to update the estimate of the distribution mean at the second stage. Along the same line, Serel (2009) explores the impact of price-sensitive demand on the retailer's ordering policy. Choi et al. (2006) extend their previous work to the case where both the mean and variance of the demand distribution are unknown, and estimated by the retailer using a two-parameter Bayesian updating method. Huang et al. (2005) investigate the retailer's optimal policy when the initial order quantity can be changed after the forecast revision by incurring both a fixed and a variable cost. Sethi et al. (2007) explore the problem in which the retailer decides the second order subject to a constraint on the service-level (probability of satisfying all demand). Our contribution to the quick response research stream described above is to include in the problem a budget constraint that limits the purchase quantities of multiple products.

The second major research stream that this paper falls into concerns the ordering policy of a multi-product newsvendor subject to a single constraint. In this well-studied problem, the newsvendor has a single ordering opportunity, and the demand distribution parameters for all products are assumed known. The constraint may arise from limited availability of resources such as budget, shelf-space, or production capacity. The newsvendor needs to determine the optimal ordering quantities of multiple products so as to maximize its total expected profit. The multiitem newsvendor problem, which dates back to Hadley and Whitin (1963), has been studied by various researchers, e.g. Nahmias and Schmidt (1984), Lau and Lau (1996), Erlebacher (2000), Moon and Silver (2000). More recently Abdel-Malek et al. (2004) develop an iterative method for solving the multi-item newsvendor problem with budget constraint. Abdel-Malek and Montanari (2005) develop a method for solving the problem with two constraints. Niederhoff (2007) uses a piecewise linear approximation method to obtain an approximate solution. Abdel-Malek and Areeratchakul (2007) propose a quadratic programming approach. Zhang et al. (2009) propose a binary solution algorithm to solve the multi-product newsvendor problem with a single constraint. Shao and Ji (2006) study a multi-item newsvendor problem with fuzzy demand. Abdel-Malek et al. (2008) study the capacitated multi-item newsboy problem with random yield. Taleizadeh et al. (2009) and Zhang (2010) investigate the optimal ordering policy when the supplier offers quantity discount. Shi and Zhang (2010) consider both supplier quantity discount and price-dependent demand. Chen and Chen (2010) study an extension where additional demand can be created by providing a price discount to customers who are willing to buy in advance. Recently, formulations of the multi-item newsboy problem with risk constraints have been proposed (Zhou et al., 2008).

Some researchers have considered the possibility of ordering additional units (emergency supply) after the demands are observed and the regular selling season ends. Morey and Sweeney (1984) study a budget-constrained multi-item procurement problem in which following the demand realization, recourse purchases can be made to reduce the level of unmet demand. They assume discrete probability distribution for demand and formulate a stochastic linear program with recourse for solving the problem. Chung et al. (2008) study a single-period multi-product production problem in which the first production batch is completed prior to demand realization. After observing the demands, production capacities previously allocated to the products can be used to reduce demand shortages. Zhang and Du (2010) study a similar multi-item single-period problem with a production capacity constraint. They include in their model both an in-house production option and an external supplier with a higher production cost and unlimited capacity.

Our work extends the traditional budget-constrained multiitem newsvendor problem to the case where there are two ordering opportunities, and the second order is placed before the selling season based on a revised demand forecast. Miltenburg and Pong (2007a, 2007b) investigate a problem which is related to the problem considered in this paper. They solve a specialized multi-item newsvendor problem with demand forecast update. There are capacity limitations associated with the two orders. They assume a two-point discrete distribution for the new demand observation and that product demands are independent. In our model we allow dependent demands, use the standard Bayesian theory, and make all purchasing decisions subject to a single budget constraint.

\section{Model}

We consider a retailer with a limited budget for purchasing multiple products that will be sold in a single selling period. The objective is to maximize the expected profit. The uncertainty in demand implies that the retailer needs to take into account the costs of overstocking and understocking. The retailer has two opportunities for buying the products. The first order must be placed at time 1 , based on a preliminary demand forecast. The order quantities for $p$ products at time 1 are shown by $Q_{1}$, $i=1, \ldots, p$. The demand forecast is revised after gathering new market information, and the retailer has a second chance to order additional units at time 2 . We use $Q_{2 i}, i=1, \ldots, p$, to denote the additional units of product $i$ ordered at time 2 . The decisions in the model are shown in Fig. 1.

The demands for products follow a multivariate normal probability distribution. The assumption of normal demand distribution is common both in practice and in the academic inventory

$$
\begin{aligned}
& \mathrm{Q}_{1 \mathrm{i}} \text { units ordered at } \\
& \text { unit purchase cost } \\
& \mathrm{c}_{1 \mathrm{i}}, i=1, \ldots, p
\end{aligned}
$$

New market

information vector $X$ is observed
Demand forecast $\quad \mathrm{Q}_{2 \mathrm{i}}$ units ordered at
is updated unit purchase cost
$\mathrm{c}_{2 \mathrm{i}}, i=1, \ldots, p$

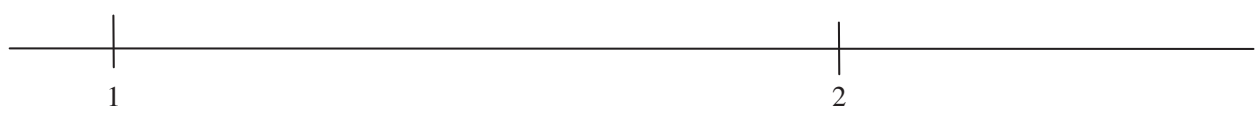

Time

Fig. 1. The ordering decisions in the model. 
literature (Silver et al., 1998; Bitran et al., 1986). The vector $M=\left(m_{1}, m_{2}, \ldots, m_{p}\right)$ represents the mean demands for $p$ products. The dependencies between the product demands at time 1 are described by the covariance matrix $\Sigma_{1}$. The elements of the $p \times p$ matrix $\Sigma_{1}$ will be referred to as $\sigma_{i j}, i=1, \ldots, p, j=1, \ldots, p$.

While we allow the mean demands $m_{1}, m_{2}, \ldots, m_{p}$ to be unknown at time 1 , we assume that the correlations between the demands, and variances of the demand distributions are known, meaning that the covariance matrix $\Sigma_{1}$ is known by the retailer. The mean demand vector $M$ itself is assumed to have a multivariate normal distribution with mean $\mu_{1}=\left(\mu_{11}, \mu_{12}, \ldots, \mu_{1 p}\right)$ and covariance matrix $D$. This distribution reflects the retailer's prior beliefs. Thus $\mu_{11}, \mu_{12}, \ldots, \mu_{1 p}$ are the initial estimates of mean demands for the products. The variances in the covariance matrix $D$ indicate how well the retailer is informed about these estimates at time 1 . A higher variance for the estimated mean of a product means that the retailer possesses less information about the average demand. The elements of the $p \times p$ matrix $D$ will be referred to as $d_{i j}, i=1, \ldots, p, j=1, \ldots, p$.

The unconditional demand distribution at time 1 is multivariate normal with mean $\mu_{1}$ and covariance matrix $\Sigma_{1}+D$. Between time 1 and time 2 the retailer collects new market information about the products. This new information is represented as a demand observation for each product. Hence a vector of demand observations $X=\left(x_{1}, x_{2}, \ldots, x_{p}\right)$ is used for revising the demand forecast at time 2 . Based on the vector $X$, a posterior probability distribution for the mean demand $M$ is formed at time 2 . We note that this modeling approach has been previously used in the literature for the single product problem (Choi et al., 2003). The model constructed here is a natural extension of that to the case of multiple products with correlated demands.

The Bayesian theory implies that the posterior distribution of the mean demand $M$ is multivariate normal with mean $\mu_{2}$ given by (Robert, 2007, p. 186)

$$
\begin{aligned}
\mu_{2}^{T} & =X^{T}-\Sigma_{1}\left(\Sigma_{1}+D\right)^{-1}\left(X-\mu_{1}\right)^{T} \\
& =\left(\Sigma_{1}^{-1}+D^{-1}\right)^{-1}\left(\Sigma_{1}^{-1} X^{T}+D^{-1} \mu_{1}^{T}\right)
\end{aligned}
$$

and covariance matrix

$D_{2}=\left(D^{-1}+\Sigma_{1}^{-1}\right)^{-1}$.

For completeness, derivation of (1) and (2) is provided in Appendix A. From (1) the random vector $\mu_{2}$ is multinormal with mean $\mu_{1}$, and covariance matrix

$V=\left(\Sigma_{1}^{-1}+D^{-1}\right)^{-1} \Sigma_{1}^{-1}\left(\Sigma_{1}+D\right)\left[\left(\Sigma_{1}^{-1}+D^{-1}\right)^{-1} \Sigma_{1}^{-1}\right]^{T}$.

Let the elements of the $p \times p$ covariance matrix $V$ be denoted as $V=\left[v_{i, j}\right]$. The predictive demand distribution at time 2 is also multivariate normal with mean $\mu_{2}$, and covariance matrix

$\Sigma_{2}=\Sigma_{1}+\left(\Sigma_{1}^{-1}+D^{-1}\right)^{-1}$.

Thus after $X$ is known, the optimal order quantities for products at time 2 can be determined based on $\mu_{2}$ and $\Sigma_{2}$. Let the elements of the $p \times p$ covariance matrix $\Sigma_{2}$ be denoted as $\Sigma_{2}=\left[a_{i, j}\right]$. Also let the elements of the mean vector $\mu_{2}$ be shown as $\mu_{2}=\left(\mu_{21}, \mu_{22}, \ldots\right.$, $\left.\mu_{2 p}\right)$. Thus the marginal demand distribution for product $i$ at time 2 is normal with mean $\mu_{2 i}$ and variance $a_{i i}, i=1,2, \ldots, p$.

Regarding the ordering decisions at time 1 , if the retailer has a budget large enough, the optimal order quantity for product $i$ at time 1 can be determined using only the marginal demand distribution for that product at time 2 . However, in the case of budget-constrained retailer, the order quantity at time 1 will depend on the multivariate probability distribution of $\mu_{2}$.

We remark that for updating the demand forecast, alternative approaches exist in the previous literature. The two-period production model of Fisher and Raman (1996) involves multiple products, of which demands in the two periods are correlated. In their approach, historical sales data for each product are used to estimate the means and variances of the marginal demand distributions as well as the correlation between them. Using this correlation estimate, the demand distribution in the second period can be written as conditional on the first period demand, yielding a more accurate demand forecast for period 2 compared to the marginal probability distribution for the second period demand. In Gurnani and Tang's (1999) single-product model, the demand and market information are assumed to be distributed as bivariate normal, and similar to Fisher and Raman (1996), the demand distribution conditional on the market information is used for deciding the purchase quantity at the second stage. In other words, the market information in the model of Gurnani and Tang (1999) plays the same role as the first period demand in Fisher and Raman (1996). In both papers, there are two different and correlated random variables associated with each product. As stated earlier, we follow the approach of Choi et al. (2003) in which there is a single random variable (with unknown mean) associated with each product. The new market information vector $X$ in our model is a draw from the joint distribution of $p$ random variables, each of which represents the seasonal demand of a particular product. This is in contrast to Gurnani and Tang (1999) in which market information is an exogenous variable.

The cost parameters and order decisions in our model are listed below

$c_{j i} \quad$ unit purchase cost for product $i$ at time $j, j=1,2, i=1,2$, ..., $p$

$\tau_{i} \quad$ unit salvage value for product $i, i=1,2, \ldots, p$

$\pi_{i} \quad$ unit shortage cost (loss of goodwill) for product $i, i=1,2$, $\ldots, p$

$p_{i} \quad$ unit selling price for product $i, i=1,2, \ldots, p$

$Q_{j i} \quad$ order quantity for product $i$ at time $j, j=1,2, i=1,2, \ldots, p$

The salvage value $\tau_{i}$ indicates the revenue from leftover stock, and the shortage cost $\pi_{i}$ is incurred when some of the demand cannot be satisfied because of insufficient stock. We assume that $\tau_{i}<c_{1 i}<c_{2 i}<p_{i}, i=1,2, \ldots, p$. If $c_{1 i} \geq c_{2 i}$, no order would be placed for item $i$ at time 1 .

\section{Multi-product problem without a budget constraint}

The two-stage multi-product problem without a budget constraint is a special case of the general problem that we consider. It can be decomposed into simpler single-product subproblems, hence, before dealing with the general case, we focus on the case of a non-binding budget constraint first.

If the retailer has a large (non-binding) budget, the optimal ordering policy for a given product can be specified similarly to the budget-unconstrained single-product problem. The earlier studies on the single-product problem have assumed that the retailer is not financially constrained (e.g., Choi et al., 2003; Huang et al., 2005).

To apply the backward dynamic programming approach, we start with considering the ordering problem at time 2 . Given that $Q_{1 i}$ units of product $i$ has been ordered at time 1 , suppose the retailer orders $Q_{2 i}$ additional units of product $i$ at time $2, i=1, \ldots, p$. Then the expected profit associated with product $i$ at time 2 can be written as

$$
\begin{aligned}
& E P_{2 i}\left(Q_{1 i}, Q_{2 i}\right)=p_{i} E\left[\min \left(Q_{1 i}+Q_{2 i}, Y_{i}\right)\right]+\tau_{i} E\left[Q_{1 i}+Q_{2 i}-Y_{i}\right]^{+} \\
& \quad-\pi_{i} E\left[Y_{i}-Q_{1 i}-Q_{2 i}\right]^{+}-c_{2 i} Q_{2 i}
\end{aligned}
$$

where $Y_{i}$ is the demand for product $i$ during the selling season, the demand distribution is normal with mean $\mu_{2 i}$ and variance $a_{i i}$, and 
$K^{+}=\max (K, 0)$. The four terms on the right side of (5) are the expected revenue, expected salvage revenue resulting from leftovers, expected shortage cost, and the purchase cost, respectively. The total expected profit function for all products at time 2 is a sum of $p$ profit functions, each of which is associated with a specific product and has the form shown in (5). Hence, to determine the optimal budget-unconstrained order quantity for a specific product at time 2 , it is sufficient to consider only the marginal demand distribution for that product. $E P_{2 i}\left(Q_{1 i}, Q_{2 i}\right)$ is concave in $Q_{2 i}$, and setting the derivative of (5) to zero, the optimal order quantity at time $2, Q_{2 i}^{*}$ is

$Q_{2 i}^{*}=\max \left\{0, \mu_{2 i}+a_{i i}^{0.5} \Phi^{-1}\left(s_{i}\right)-Q_{1 i}\right\}$,

where $\Phi^{-1}\left(s_{i}\right)$ is the inverse of the standard normal cumulative distribution function (cdf). The threshold $s_{i}$ is the critical percentile point of the demand distribution that balances the tradeoffs between the underage and overage costs in the standard newsvendor problem, i.e.,

$s_{i}=\frac{p_{i}+\pi_{i}-c_{2 i}}{p_{i}+\pi_{i}-\tau_{i}}$

Thus the retailer will order additional units of product $i$ if the updated mean $\mu_{2 i}$ is greater than $Q_{1 i}-a_{i i}^{0.5} \Phi^{-1}\left(s_{i}\right)$. Let $t_{i}=\mu_{2 i}+a_{i i}^{0.5} \Phi^{-1}\left(s_{i}\right)$. If $Q_{2 i}^{*}>0$, the retailer's expected profit at time $2, J_{1 i}\left(Q_{1 i}, \mu_{2 i}\right)$, will be

$$
\begin{aligned}
J_{1 i}\left(Q_{1 i}, \mu_{2 i}\right)= & p_{i} E\left[\min \left(t_{i}, Y_{i}\right)\right]+\tau_{i} E\left[t_{i}-Y_{i}\right]^{+}-\pi_{i} E\left[Y_{i}-t_{i}\right] \\
& -c_{2 i}\left(t_{i}-Q_{1 i}\right)=\left(p_{i}-c_{2 i}\right) \mu_{2 i}+\left(\tau_{i}-c_{2 i}\right) a_{i i}^{0.5} \Phi^{-1}\left(s_{i}\right) \\
& -\left(p_{i}+\pi_{i}-\tau_{i}\right) a_{i i}^{0.5} \Psi\left(\Phi^{-1}\left(s_{i}\right)\right)+c_{2 i} Q_{1 i},
\end{aligned}
$$

where $\Psi(u)=\int_{u}^{\infty}(z-u) \varphi(z) d z$ is the unit loss function for the standard normal distribution, and $\varphi(z)$ is the density of a standard normal variable. If $Q_{2 i}^{*}=0$, the retailer's expected profit, $J_{2 i}$ $\left(Q_{1 i}, \mu_{2 i}\right)$, is

$$
\begin{aligned}
J_{2 i}\left(Q_{1 i}, \mu_{2 i}\right) & =p_{i} E\left[\min \left(Q_{1 i}, Y_{i}\right)\right]+\tau_{i} E\left[Q_{1 i}-Y_{i}\right]^{+}-\pi_{i} E\left[Y_{i}-Q_{1 i}\right]^{+} \\
& =p_{i} \mu_{2 i}+\tau_{i}\left(Q_{1 i}-\mu_{2 i}\right)-\left(p_{i}+\pi_{i}-\tau_{i}\right) a_{i i}^{0.5} \Psi\left(\frac{Q_{1 i}-\mu_{2 i}}{a_{i i}^{0.5}}\right) .
\end{aligned}
$$

Combining the two cases, $Q_{2 i}^{*}>0$ and $Q_{2 i}^{*}=0$, the retailer's expected profit associated with product $i$ at time $1, E P_{1 i}\left(Q_{1 i}\right)$, can be written as

$$
\begin{aligned}
E P_{1 i}\left(Q_{1 i}\right)= & \int_{-\infty}^{\infty} E P_{2 i}\left(Q_{1 i}, Q_{2 i}^{*}\right) g_{i}\left(\mu_{2 i}\right) d \mu_{2 i}-c_{1 i} Q_{1 i} \\
= & \int_{-\infty}^{Q_{1 i}-a_{i i}^{0.5} \Phi^{-1}\left(s_{i}\right)} J_{2 i}\left(Q_{1 i}, \mu_{2 i}\right) g_{i}\left(\mu_{2 i}\right) d \mu_{2 i} \\
& +\int_{Q_{1 i}-a_{i i}^{0.5} \Phi^{-1}\left(s_{i}\right)}^{\infty} J_{1 i}\left(Q_{1 i}, \mu_{2 i}\right) g_{i}\left(\mu_{2 i}\right) d \mu_{2 i}-c_{1 i} Q_{1 i} .
\end{aligned}
$$

The probability density function (pdf) of $\mu_{2 i}$ in (8), $g_{i}\left(\mu_{2 i}\right)$, is normal with mean $\mu_{1 i}$ and variance $v_{i i}$. Thus the retailer's expected profit at time 1 in the budget-unconstrained multi-product problem is

$E P\left(Q_{11}, Q_{12}, \ldots, Q_{1 p}\right)=\sum_{i=1}^{p} E P_{1 i}\left(Q_{1 i}\right)$.

It can be shown that $E P\left(Q_{11}, Q_{12}, \ldots, Q_{1 p}\right)$ is concave in the firststage order quantities $Q_{11}, Q_{12}, \ldots, Q_{1 p}$.

Proposition 1. The retailer's expected profit in the budget-unconstrained multi-product problem is concave in the variables $Q_{1 i}, i=1$, $2, \ldots, p$.

The proofs of all propositions can be found in Appendix A. Based on Proposition 1, the optimal order quantity for product $i$ at time 1 follows from the first-order optimality condition. Using (8), the first derivative of $E P_{1 i}\left(Q_{1 i}\right)$ is

$$
\begin{aligned}
& \frac{\partial E P_{1 i}\left(Q_{1 i}\right)}{\partial Q_{1 i}}=\left(p_{i}+\pi_{i}-c_{2 i}\right) \Phi\left(\varepsilon_{i}\right)+\left(c_{2 i}-c_{1 i}\right) \\
& -\left(p_{i}+\pi_{i}-\tau_{i}\right) \int_{-\infty}^{\kappa_{i}} \Phi\left(\frac{Q_{1 i}-\mu_{1 i}-\gamma_{i} v_{i i}^{0.5}}{a_{i i}^{0.5}}\right) \varphi\left(\gamma_{i}\right) d \gamma_{i}
\end{aligned}
$$

where $\Phi(\cdot)$ is the standard normal cdf, $\kappa_{i}=Q_{1 i}-a_{i i}^{0.5} \Phi^{-1}\left(s_{i}\right)$, $\gamma_{i}=\left(\left(\mu_{2 i}-\mu_{1 i}\right) / v_{i i}^{0.5}\right)$, and $\varepsilon_{i}=\left(\left(Q_{1 i}-a_{i i}^{0.5} \Phi^{-1}\left(s_{i}\right)-\mu_{1 i}\right) / v_{i i}^{0.5}\right)$, cf. Choi et al. (2003, Lemma 1 ). The optimal $Q_{11}$, if positive, satisfies the equation $\left(\partial E P_{1 i}\left(Q_{1 i}\right)\right) /\left(\partial Q_{1 i}\right)=0$. To find the optimal set of initial order quantities $Q_{1 i}, i=1, \ldots, p$, it is sufficient to solve $p$ singleproduct problems separately. When the product demands are correlated with each other, the optimal order quantity $Q_{1 i}$ for product $i$ found by using (10) depends on the demand distributions of other products. In the special case when the demands for products are statistically independent, the first-order optimality condition for a product will depend on only the demand and cost parameters for that product, and the optimal order at time 1 will be exactly same as that derived in the single-product problem of Choi et al. (2003). On the other hand, the multi-product problem under a binding budget constraint is not separable into singleproduct problems, and the optimal initial orders for the products must be determined jointly.

\section{Single product problem with a budget constraint}

We now consider the single-product problem with demand forecast update and a budget constraint. In the presence of a budget constraint, the problem involves determining how much of the available funds to reserve for purchases at the second stage. Let $B$ be the total budget available for purchases at time 1 and time 2 combined. Since there is only a single product, we omit the subscript for the product number in the notation in this section.

After the forecast update, the target inventory at time 2 is $\mu_{2}+\Phi^{-1}(s) \sigma_{x}$ where $\sigma_{x}$ is the standard deviation of the predictive demand distribution at time 2 . We note that $\sigma_{x}$ corresponds to $a_{i i}^{0.5}$ for product $i$ that has an independent demand distribution in the multi-product problem (see e.g., Serel, 2009). We can write the order amount at time $2, Q_{2}$, as

$$
\begin{aligned}
Q_{2} & =\max \left\{0, \mu_{2}+\Phi^{-1}(s) \sigma_{x}-Q_{1}\right\} \quad \text { if } \quad c_{2}\left[\mu_{2}+\Phi^{-1}(s) \sigma_{x}-Q_{1}\right]<B-c_{1} Q_{1}, \\
& =\max \left\{0,\left(B-c_{1} Q_{1}\right) / c_{2}\right\} \quad \text { if } \quad c_{2}\left[\mu_{2}+\Phi^{-1}(s) \sigma_{x}-Q_{1}\right] \geq B-c_{1} Q_{1}
\end{aligned}
$$

The amount of money on hand at time 2 is $B-c_{1} Q_{1}$. If there are sufficient funds at time 2 , the retailer raises the inventory on hand to the target level $\mu_{2}+\Phi^{-1}(s) \sigma_{x}$. Otherwise, all funds available are used to bring the inventory to the target level as close as possible.

The ordering policy at time 2 can be expressed in terms of three regions for the updated mean estimate $\mu_{2}$

$$
Q_{2}=\left[\begin{array}{lll}
0 & \text { if } & \mu_{2} \leq Q_{1}-\Phi^{-1}(s) \sigma_{x}, \\
\mu_{2}+\Phi^{-1}(s) \sigma_{x}-Q_{1} & \text { if } & Q_{1}-\Phi^{-1}(s) \sigma_{x}<\mu_{2} \leq Q_{1}-\Phi^{-1}(s) \sigma_{x}+\frac{B-c_{1} Q_{1}}{c_{2}}, \\
\frac{B-c_{1} Q_{1}}{c_{2}} & \text { if } & \mu_{2}>Q_{1}-\Phi^{-1}(s) \sigma_{x}+\frac{B-c_{1} Q_{1}}{c_{2}} .
\end{array}\right.
$$

Let $W=Q_{1}\left(1-\left(c_{1} / c_{2}\right)\right)+\left(B / c_{2}\right)$. The expected profit at time 2 can be written as a function of $Q_{1}$ and $\mu_{2}$ as follows:

Case 1. If $\mu_{2} \leq Q_{1}-\Phi^{-1}(s) \sigma_{x}$, the total inventory at the beginning of the season will be $Q_{1}$, and the expected profit $T_{1}\left(Q_{1}, \mu_{2}\right)$ is

$T_{1}\left(Q_{1}, \mu_{2}\right)=p \mu_{2}+\tau\left(Q_{1}-\mu_{2}\right)-(p+\pi-\tau) \sigma_{x} \Psi\left(\frac{Q_{1}-\mu_{2}}{\sigma_{x}}\right)$.

Case 2. If

$Q_{1}-\Phi^{-1}(s) \sigma_{x}<\mu_{2} \leq Q_{1}-\Phi^{-1}(s) \sigma_{x}+\left(\left(B-c_{1} Q_{1}\right) / c_{2}\right)$, 
we have $Q_{1}+Q_{2}=\mu_{2}+\Phi^{-1}(s) \sigma_{x}$ and the expected profit $T_{2}\left(Q_{1}, \mu_{2}\right)$ is

$T_{2}\left(Q_{1}, \mu_{2}\right)=\left(p-c_{2}\right) \mu_{2}+\left(\tau-c_{2}\right) \sigma_{x} \Phi^{-1}(s)-(p+\pi-\tau) \sigma_{x} \Psi\left(\Phi^{-1}(s)\right)+c_{2} Q_{1}$.

Case 3. If $\mu_{2}>Q_{1}-\Phi^{-1}(s) \sigma_{x}+\left(\left(B-c_{1} Q_{1}\right) / c_{2}\right)$, the total inventory is $W$, the expected profit $T_{3}\left(Q_{1}, \mu_{2}\right)$ is

$T_{3}\left(Q_{1}, \mu_{2}\right)=p \mu_{2}+\tau\left(W-\mu_{2}\right)-(p+\pi-\tau) \sigma_{x} \Psi\left(\frac{W-\mu_{2}}{\sigma_{x}}\right)-\left(B-c_{1} Q_{1}\right)$.

By taking expectation with respect to $\mu_{2}$, the expected profit at time $1, E P\left(Q_{1}\right)$, can be written as

$$
\begin{gathered}
E P\left(Q_{1}\right)=\int_{-\infty}^{Q_{1}-\sigma_{x} \Phi^{-1}(s)} T_{1}\left(Q_{1}, \mu_{2}\right) g\left(\mu_{2}\right) d \mu_{2}+\int_{Q_{1}-\sigma_{\chi} \Phi^{-1}(s)}^{Q_{1}-\sigma_{\chi} \Phi^{-1}(s)+\left(\left(B-c_{1} Q_{1}\right) / c_{2}\right)} \\
T_{2}\left(Q_{1}, \mu_{2}\right) g\left(\mu_{2}\right) d \mu_{2}+\int_{Q_{1}-\sigma_{\chi} \Phi^{-1}(s)+\left(\left(B-c_{1} Q_{1}\right) / c_{2}\right)}^{\infty} \\
T_{3}\left(Q_{1}, \mu_{2}\right) g\left(\mu_{2}\right) d \mu_{2}-c_{1} Q_{1},
\end{gathered}
$$

where $g\left(\mu_{2}\right)$ is the pdf of $\mu_{2}$. In Proposition 2, we show that $E P\left(Q_{1}\right)$ is concave in $Q_{1}$.

Proposition 2. The retailer's expected profit in the budget-constrained single-product problem is concave in the initial order $Q_{1}$.

Thus, the optimal first-stage order quantity in the budgetconstrained single-product problem can be found by equating the first derivative of $E P\left(Q_{1}\right)$ to zero (Eq. (A10) in Appendix A). In Proposition 3 , we show that as the budget increases, the expected profit increases at a decreasing rate.

Proposition 3. The retailer's expected profit in the budget-constrained single-product problem is non-decreasing concave in the budget amount $B$.

Let $Q_{1}^{u}$ be the optimal first-stage order quantity, and $c_{1} Q_{1}^{u}$ be the total procurement cost at the first-stage in the budgetunconstrained single-product problem. It can be shown that when the budget available exceeds the threshold $c_{1} Q_{1}^{u}$, the optimal initial order in the unconstrained-budget problem is a lower bound for the optimal initial order in the constrainedbudget problem.

Proposition 4. The retailer's optimal initial order in the budgetconstrained single-product problem is greater than or equal to $Q_{1}^{u}$ when the budget B exceeds $c_{1} Q_{1}^{u}$.

The optimal first-stage order quantity in the budget-constrained problem depends on the budget amount in the following manner. When the budget available $B$ is less than $c_{1} Q_{1}^{u}$, the optimal first-stage order quantity equals $B / c_{1}$; the retailer purchases as much as it can at time 1 . However, when the budget available exceeds $c_{1} Q_{1}^{u}$, it does not imply that the optimal firststage order quantity will be $Q_{1}^{u}$. Given a limited budget, the retailer takes advantage of the relatively lower purchase cost at time 1 , and orders more than $Q_{1}^{u}$ at time 1 . The amount of money left for additional purchases at time 2 determines how many extra units the retailer will add to the inventory above $Q_{1}^{u}$ at time 1 . If the total funds are tight and expected to be spent fully before the season, it may be preferable to order at time 1 rather than at time 2 due to the savings in the unit purchase cost. By ordering most of the stock at time 1 , the retailer is able to increase its stocking level and reduce the risk of unsatisfied demand. On the other hand, when the budget available becomes sufficiently large, there are enough funds for possible purchases at time 2 . As the budget available $B$ goes to infinity, the right side of (A26) approaches to the right side of (A25), and the first derivative of the expected profit becomes the same for both the budget-unconstrained and budget-constrained single-product problems. The retailer does not face the risk of insufficient funds at time 2 , and the order size at time 1 converges to the order quantity in the budget-unconstrained problem $Q_{1}^{u}$.

We note that the optimal order quantity in a budget-unconstrained single-stage problem is an upper bound on the optimal order quantity in the budget-constrained single-stage problem. We have shown that this property does not extend to the initial order quantity in the two-stage problem.

It can also be shown that the optimal initial order is nondecreasing in the second-stage purchase cost $c_{2}$.

Proposition 5. The retailer's optimal initial order in the budgetconstrained single-product problem is non-decreasing in the secondstage purchase cost $c_{2}$.

\section{Multi-product problem with a budget constraint}

We now analyze the most complicated case, that is, there are multiple products and the funds available for purchase are tight. As in the cases considered previously, dynamic programming approach is used. We first solve the second stage problem given the ordering decisions at the first stage, and substitute this solution into the expected profit function at stage 1 to find the optimal stage 1 decisions. We use $B$ to denote the total funds available for all products.

Let $Q_{1}=\left(Q_{11}, Q_{12}, \ldots, Q_{1 p}\right)$ and $Q_{2}=\left(Q_{21}, Q_{22}, \ldots, Q_{2 p}\right)$ be the vectors that contain the purchase quantities at time 1 and time 2 , respectively. The retailer's problem at time 2 is essentially a constrained multi-product newsvendor problem with initial inventories $Q_{1 i}, i=1, \ldots, p$, and an available budget of $B-\sum_{i=1}^{p} c_{1 i} Q_{1 i}$. Given $Q_{1 i}, i=1, \ldots, p$, units that have been ordered at time 1 , and the market information $X$, the optimal order quantities $Q_{2 i}, i=1, \ldots, p$, at time 2 can be found by solving the following optimization problem:

$$
\begin{aligned}
\text { Maximize } B_{2}\left(Q_{1}, Q_{2}, \mu_{2}\right)= & \sum_{i=1}^{p} p_{i} E\left[\min \left(Q_{1 i}+Q_{2 i}, Y_{i}\right)\right]+\tau_{i} E\left[Q_{1 i}+Q_{2 i}-Y_{i}\right]^{+} \\
& -\pi_{i} E\left[Y_{i}-Q_{1 i}-Q_{2 i}\right]^{+}-c_{2 i} Q_{2 i} \\
\text { subject to } \sum_{i=1}^{p} c_{2 i} Q_{2 i} \leq & B-\sum_{i=1}^{p} c_{1 i} Q_{1 i} .
\end{aligned}
$$

The objective function $B_{2}\left(Q_{1}, Q_{2}, \mu_{2}\right)$ is concave in the decision variables $Q_{2 i}, i=1, \ldots, p$, and using Karush-Kuhn-Tucker (KKT) conditions, the optimal order quantities, if positive, are given by

$$
\begin{aligned}
& Q_{2 i}^{*}=F_{i}^{-1}\left(\frac{p_{i}+\pi_{i}-c_{2 i}-\lambda c_{2 i}}{p_{i}+\pi_{i}-\tau_{i}}\right)-Q_{1 i}, \\
& \lambda\left[B-\sum_{i=1}^{p}\left(c_{1 i} Q_{1 i}+c_{2 i} Q_{2 i}^{*}\right)\right]=0 .
\end{aligned}
$$

where $\lambda$ is the Lagrange multiplier, $\lambda \geq 0$, and $F_{i}$ is the cdf of the demand at time 2 for product $i$, i.e., a normal distribution with mean $\mu_{2 i}$ and variance $a_{i i}$. The optimal values for $Q_{2 i}$ satisfying (17) can be found using one of the algorithms available for solving the constrained multi-product newsvendor problem, e.g. AbdelMalek et al. (2004) and Zhang et al. (2009).

Let $\kappa\left(Q_{1}, \mu_{2}\right)$ be the optimal value of $B_{2}\left(Q_{1}, Q_{2}, \mu_{2}\right)$ in problem (16). The retailer's expected profit at time 1 can be written as

$E P\left(Q_{1}\right)=E_{\mu_{2}} \kappa\left(Q_{1}, \mu_{2}\right)-\sum_{i=1}^{p} c_{1 i} Q_{1 i}$

It is stated in Proposition 6 that the expected profit at time 1 is jointly concave in the decision variables $Q_{1 i}, i=1, \ldots, p$. 
Proposition 6. The retailer's expected profit in the budget-constrained multi-product problem is jointly concave in the initial order quantities $Q_{1 i}, i=1, \ldots, p$.

To determine the optimal ordering decisions at time 1 , a computational procedure can be used. Suppose we discretize the $p$-dimensional grid that contains all possible values for the $p$-dimensional vector $\mu_{2}=\left(\mu_{21}, \mu_{22}, \ldots, \mu_{2 p}\right)$. Given a particular point on this grid $\mu_{2}=\mu_{2}^{a}$, and the initial orders $Q_{1 i}, i=1, \ldots, p$, the retailer's problem at time 2 is defined by (16). The demands for products in this problem are distributed as multinormal with mean $\mu_{2}^{a}$ and covariance matrix $\Sigma_{2}$. After solving (16) based on $\mu_{2}=\mu_{2}^{a}$, by subtracting the cost of purchases at time 1 from the stage 2 profit associated with optimal $Q_{2}$, we can find the retailer's profit at time 1 given $Q_{1 i}$ and a particular realization for $\mu_{2}, \mu_{2}^{a}$.

The retailer's expected profit at time 1 for a given $Q_{1 i}$ and prior to observing the market information $X$ can be computed using the joint probability distribution of predictive demand mean, $\mu_{2}$. Let $\eta\left(\mu_{2}\right)$ be the joint density of the random variables $\mu_{21}, \mu_{22}, \ldots, \mu_{2 p}$. Recall that $\mu_{21}, \mu_{22}, \ldots, \mu_{2 p}$ are distributed multinormal with mean $\mu_{1}$ and covariance matrix $V$. For example, if there are two

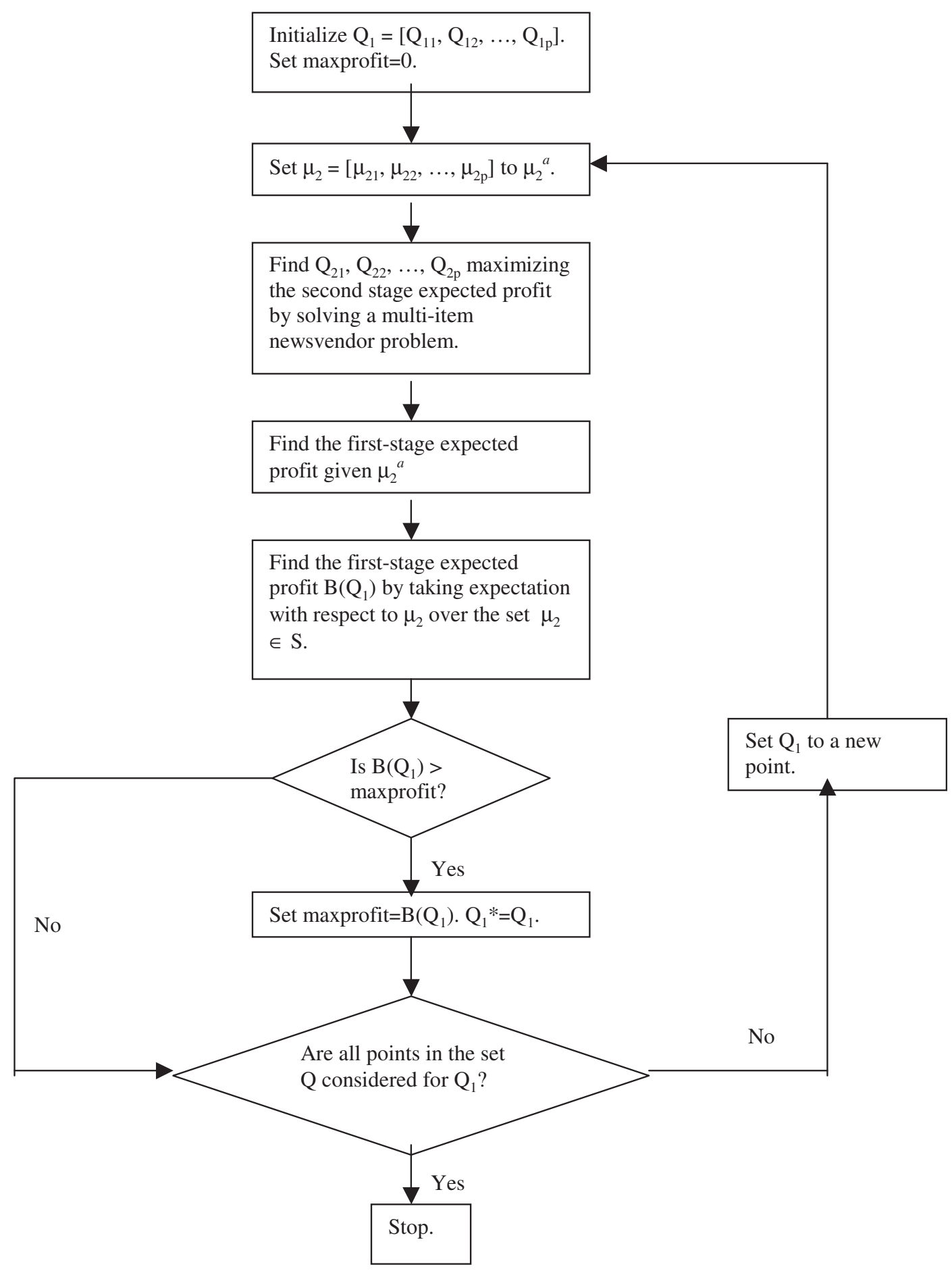

Fig. 2. Solution procedure for the budget-constrained multi-product problem. 
products (i.e., $p=2$ ), the joint pdf of $\mu_{21}$ and $\mu_{22}$ is bivariate normal given by

$\eta\left(\mu_{21}, \mu_{22}\right)=\frac{1}{2 \pi \sqrt{v_{11} v_{22}\left(1-\rho^{2}\right)}} \exp \left[-\left(\frac{y_{1}^{2}-2 \rho y_{1} y_{2}+y_{2}^{2}}{2\left(1-\rho^{2}\right)}\right)\right]$,

where $y_{1}=\left(\mu_{21}-\mu_{11}\right) / v_{11}^{0.5}, \quad y_{2}=\left(\mu_{22}-\mu_{12}\right) / v_{22}^{0.5}, \quad$ and $\rho=v_{12} /$ $\sqrt{v_{11} v_{22}}$. In general, for $p \geq 2$ we have (see, e.g., Robert, 2007, p. 519)

$\eta\left(\mu_{2}\right)=\frac{1}{(2 \pi)^{0.5 p} \sqrt{\operatorname{det}(V)}} \exp \left[-0.5\left(\mu_{2}-\mu_{1}\right) V^{-1}\left(\mu_{2}-\mu_{1}\right)^{T}\right]$.

If $B\left(Q_{11}, Q_{12}, \ldots, Q_{1 p} \mid \mu_{2}\right)$ is the expected profit for a given realization of $\mu_{2}$, the expected profit at time 1 prior to the realization of $\mu_{2}$ is

$E P\left(Q_{11}, Q_{12}, \ldots, Q_{1 p}\right)=\iint \ldots \int B\left(Q_{11}, Q_{12}, \ldots, Q_{1 p} \mid \mu_{2}\right) \eta\left(\mu_{2}\right) d \mu_{21} d \mu_{22} \ldots d \mu_{2 p}$.

By suitably discretizing $\eta\left(\mu_{2}\right)$ over the region for $\mu_{2}$ that contains all possible values of $\mu_{2}$ for practical purposes, we can calculate the expected profit at time 1 for a particular choice of $Q_{1 i}, i=1, \ldots$, $p$. The idea is to approximate the integral representing the expected profit by a weighted sum of values which are derived by evaluating the term inside the integral at a discrete set of points. If $S$ is the set of points for $\mu_{2}$ at which the retailer's profit is calculated, the retailer's expected profit at time 1 can be expressed as

$B\left(Q_{11}, Q_{12}, \ldots, Q_{1 p}\right)=\sum_{\mu_{2} \in S} B\left(Q_{11}, Q_{12}, \ldots, Q_{1 p} \mid \mu_{2}\right) \eta\left(\mu_{2}\right)$.

To calculate the expected profit, we evaluate (21) at all possible combinations of the specific values for components $\mu_{2 i}$ of the vector $\mu_{2}$. The consecutive points for each component $\mu_{2 i}$ are specified along an interval starting at the lowest value $\mu_{2 i}=2.5$ with a step size of 1 up to the maximum value of $\mu_{1 i}+3.5 v_{i i}^{0.5}$. The probability that $\mu_{2 i}$ exceeds the higher limit of the interval is considered to be negligible, and hence we ignore the contribution to the expected profit when $\mu_{2 i}>\mu_{1 i}+3.5 v_{i i}^{0.5}$. Because we use a step size of 1 for all $p$ dimensions, the weight applied to each term that enters the sum (and evaluated at a particular $\mu_{2}$ ) in (21) is 1 . In the special case of one dimension $(p=1)$, the pdf of $\mu_{2}$ evaluated at a particular value of $\mu_{2}$, say $\mu_{2}^{a}$, is multiplied by 1 , which is approximately the area of the strip under the pdf between $\mu_{2}^{a}-0.5$ and $\mu_{2}^{a}+0.5$. To understand the rationale of (21), it can be thought that when there are $p$ dimensions, we have $p$ nested integrals on the right side of (20) and we evaluate these nested integrals iteratively. Starting with the innermost layer, we evaluate the integral for a single dimension, and substitute this evaluation into the integral immediately nesting it. Thus using a step size of 1 for all $p$ dimensions, (21) provides an approximation to the evaluation of the $p$-dimensional integral that represents the expected profit, (20).

Note that to limit the number of points used in the computations, the units in which demand is measured may need to be redefined (i.e., scaled down) if the mean demand $\mu_{1 i}$ is significantly large. Another alternative to approximate the multi-dimensional integral yielding the expected profit is to use quadrature methods such as Gauss-Hermite instead of (21) (e.g., Press et al., 1992).

Finally, the optimal set of decisions at time 1 can be found using a grid search. After computing $B\left(Q_{11}, Q_{12}, \ldots, Q_{1 p}\right)$ for all possible combinations of $Q_{11}, Q_{12}, \ldots, Q_{1 p}$, we can determine the optimal combination of $Q_{11}, Q_{12}, \ldots, Q_{1 p}$ that maximizes the retailer's expected profit at time 1 . Let $Q$ be the set of points over which the search for the optimal initial orders is conducted. The procedure described above is summarized in a flow-chart in Fig. 2. The output of the algorithm are $Q_{1}^{*}$, the vector containing the optimal values of $Q_{11}, Q_{12}, \ldots, Q_{1 p}$, and maxprofit, the optimal expected profit at time 1 . Note that the full grid (solution space) spanning all discrete values of $Q_{11}, Q_{12}, \ldots, Q_{1 p}$ does not need to be searched. Given a set of values $Q_{11}, Q_{12}, \ldots, Q_{1 i-1}, Q_{1 i+1}, \ldots, Q_{1 p}$, to find the best value for $Q_{1}$, the search can be started at the lowest value specified for $Q_{1 i}$ and the value of $Q_{1 i}$ is increased by the step size used, say $\Delta Q_{1 i}$, at each iteration until the expected profit starts decreasing. Due to the concavity of the expected profit function by Proposition 6 , the $Q_{1 i}$ values falling in the remaining unexplored high-end region cannot yield a better profit, and hence do not need to be considered. Note also that when the number of products is large, instead of grid search, with appropriate modifications well-known numerical optimization techniques such as Nelder-Mead downhill simplex method can be applied to find the optimal ordering policy (e.g. Press et al., 1992).

To check the accuracy of our approximation formula (21), in our numerical study in Section 7, we conducted Monte Carlo simulation to evaluate the expected profit at the optimal point that is found using (21). In the simulation, expected profit function (20) was computed by generating random vectors from the multivariate normal distribution for $\mu_{2}$. In the numerical examples considered, the observed maximum difference between the expected profits calculated via (21) and simulation was less than $0.4 \%$.

When the budget available is sufficiently large, the optimal first-stage order $Q_{1}^{*}$ can be approximated by the optimal order sizes in the budget-unconstrained problem, which can be determined using (10).

It is possible to find an upper bound on the optimal initial order for a product by considering the budget-unconstrained single-stage problem. Suppose the retailer does not have the option to revise the demand forecast and issue a second order. In this single-stage traditional newsvendor problem, the retailer orders only once based on the demand forecast at time 1 . The solution to this problem is an upper bound on the optimal initial order in the two-stage multi-product problem. Thus, the upper bound on the optimal $Q_{1 i}$ is given by

$Q_{1 i}^{u b}=\mu_{1 i}+\left(d_{i i}+\sigma_{i i}\right)^{0.5} \Phi^{-1}\left(\frac{p_{i}+\pi_{i}-c_{1 i}}{p_{i}+\pi_{i}-\tau_{i}}\right), \quad i=1, \ldots, p$.

In the two-stage problem, due to the possibility of learning a lower expected demand from the market signal, the retailer orders less than $Q_{1 i}^{u b}$ units of product $i$ at time 1 . Note that besides the demand learning factor, existence of a budget constraint also may induce the retailer to reduce its order size. The upper bounds given by (22) can be used to define the search region for $Q_{1 i}$ in the algorithm described in Fig. 2.

We also remark that in a related problem, instead of a common budget, there may be $p$ different budgets $B_{1}, B_{2}, \ldots, B_{p}$ specifically reserved for each product. The solution to this problem can be found by solving $p$ constrained single-product problems separately, as discussed in Section 5.

\section{Numerical examples}

In this section we present numerical examples for the models developed in the previous sections. We investigate how the optimal ordering policy is influenced by the changes in the available budget and demand uncertainty.

\subsection{Examples for the budget-constrained single-product problem}

In the first set of examples we focus on the budget-constrained single-product problem assuming that demand distribution at time 1 is normal with unknown mean $m$ and known variance $\sigma_{1}^{2}$. The mean of the demand distribution, $m$ itself is assumed to be 
Table 1

Optimal first-stage order, expected purchase cost, percentage of purchase cost incurred at time 1 , and expected profit for the budget-constrained single-product problem ( $\left.c_{1}=5, c_{2}=7, \tau=1, p=20, \pi=2, \mu_{1}=20, \sigma_{1}^{2}=2\right)$.

\begin{tabular}{rrrrrr}
\hline$d_{1}$ & \multicolumn{1}{c}{ B } & $Q_{1}^{c}$ & EPC & $r(\%)$ & $E P\left(Q_{1}^{c}\right)$ \\
\hline \multirow{2}{*}{10} & 90 & 18.0 & 90.0 & 100 & 253.3 \\
& 100 & 20.0 & 100.0 & 100 & 271.0 \\
& 110 & 22.0 & 110.0 & 100 & 279.3 \\
& 120 & 21.5 & 111.5 & 96.4 & 282.1 \\
& 130 & 21.1 & 112.5 & 93.8 & 283.4 \\
20 & 140 & 21.0 & 113.0 & 92.9 & 284.0 \\
& 100 & 18.0 & 90.0 & 100 & 244.2 \\
& 110 & 20.0 & 100.0 & 100 & 260.7 \\
& 120 & 22.0 & 110.0 & 100 & 270.2 \\
& 130 & 21.8 & 113.0 & 96.5 & 274.5 \\
& 140 & 20.9 & 114.4 & 93.1 & 277.4 \\
& & 115.2 & 90.7 & 279.2 \\
\hline
\end{tabular}

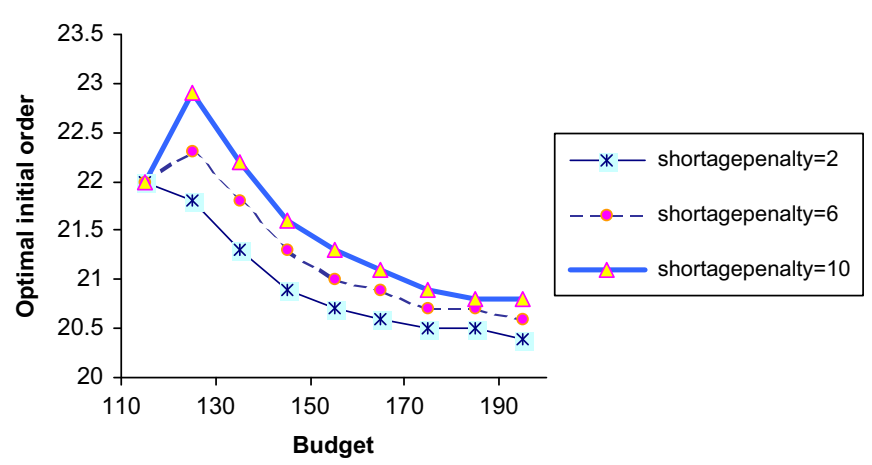

Fig. 3. Effect of shortage penalty $\pi$ on optimal initial order at different budget levels in the single-product problem.

normally distributed with mean $\mu_{1}$ and variance $d_{1}$. In our study we use the following values for parameters: $\tau=1, \pi=2, p=20$, $\sigma_{1}^{2}=2, \mu_{1}=20, d_{1} \in\{10,20\}$. The unit purchase costs at time 1 and time 2 are $c_{1}=5, c_{2}=7$, respectively.

The optimal first-stage order $Q_{1}^{c}$ and the resulting expected profit at time $1, E P\left(Q_{1}^{c}\right)$, associated with different budget values $B$ are shown in Table 1 . A higher value for $d_{1}$ indicates a more variable demand distribution. The optimal budget-unconstrained first-stage order $Q_{1}^{u}$ is 20.9 and 20.4 when $d_{1}$ is 10 and 20 , respectively. The optimal expected profit in the budget-unconstrained problem is 284.2 and 281.2 when $d_{1}$ is 10 and 20 , respectively.

The changes in expected profit values in Table 1 indicate that, in consistence with Proposition 3, marginal benefit of additional budget decreases as the budget becomes larger. Comparing the results for $d_{1}=10$ and $d_{1}=20$ in Table 1 , we observe that the expected profit decreases with demand variability. The optimal first-stage order size varies with the budget available. As noted earlier, when the budget $B$ is neither too small nor large, the firststage order exceeds the first-stage order in the unconstrainedbudget case. Table 1 shows that when the budget is small, the best decision is to spend all funds at time 1 , and hence, the firststage order $Q_{1}^{c}$ is not affected by demand variability in this case.

We also list in Table 1 the total expected purchase cost, EPC, and the percentage of this cost incurred at time $1, r$. The difference between the purchase cost and the salvage value represents the holding cost for the retailer. Hence, the unit holding cost resulting from a stock purchased at time 2 is higher than that purchased at time 1 . The values for $r$ in Table 1 are generally high, indicating that a substantial portion of the procurement funds are spent at time 1 . Thus, in our example benefit from a lower holding cost dominates the benefit from improved demand forecast at time 2 .
Table 2

Optimal order quantity and expected profit for the single-stage problem with parameters given in Table 1.

\begin{tabular}{rrrr}
\hline$d_{1}$ & $\mathrm{~B}$ & $Q_{1}^{\text {ss }}$ & $E P\left(Q_{1}^{s S}\right)$ \\
\hline 10 & 90 & 18.0 & 253.3 \\
& 100 & 20.0 & 271.0 \\
& 110 & 22.0 & 279.3 \\
& 120 & 23.0 & 280.2 \\
& 130 & 23.0 & 280.2 \\
& 140 & 23.0 & 280.2 \\
& 90 & 18.0 & 244.2 \\
20 & 100 & 20.0 & 260.7 \\
& 110 & 22.0 & 270.2 \\
& 120 & 24.0 & 273.2 \\
& 130 & 24.1 & 273.2 \\
& 140 & 24.1 & 273.2 \\
\hline
\end{tabular}

Table 3

Cost and demand parameters in the numerical example of products with independent demands.

\begin{tabular}{lllllllll}
\hline Product $(i)$ & $\tau_{i}$ & $\pi_{i}$ & $c_{1 i}$ & $c_{2 i}$ & $p_{i}$ & $\mu_{1 i}$ & $\sigma_{i i}$ & $d_{i i}$ \\
\hline 1 & 1 & 2 & 5 & 7 & 20 & 20 & 2 & 20 \\
2 & 1 & 2 & 5 & 7 & 20 & 20 & 2 & 10 \\
3 & 1 & 2 & 5 & 10 & 20 & 20 & 2 & 20 \\
\hline
\end{tabular}

The impact of shortage penalty cost $\pi$ on the optimal initial order quantity $Q_{1}^{c}$ at various budget levels is displayed in Fig. 3. The results pertain to the case $d_{1}=20$. It can be observed from Fig. 3 that when the budget exceeds a threshold level, the optimal initial order increases as $\pi$ increases.

For comparison purposes, we present in Table 2 the optimal solution for the single-stage problem. As indicated by (22), if the budget is sufficient, the optimal order quantity for the singlestage problem, $Q_{1}^{s s}$, is given by

$Q_{1}^{S S}=\mu_{1}+\left(d_{1}+\sigma_{1}^{2}\right)^{0.5} \Phi^{-1}\left(\frac{p+\pi-c_{1}}{p+\pi-\tau}\right)$.

If the budget $B$ is not sufficient to purchase $Q_{1}^{s s}$ units, all money available will be spent, and the optimal order size will be $B / c_{1}$. The difference between the profits in Tables 1 and 2 indicates the value of implementing a quick response system over using the traditional single order approach. The results indicate that the benefit from placing two distinct orders increases with the available budget. When the funds are very tight, the retailer exhausts all of its budget at time 1 even though it has a chance to order again later. Thus, a limited budget blurs the distinction between the two-stage problem and the single-stage problem. Note also that in the unconstrained-budget case, as expected, the order size in the single-stage problem exceeds the initial order size in the two-stage problem. When a second order opportunity is not available, the retailer stocks more units at time 1 .

\subsection{Multi-product problem with independent demands}

We now turn our attention to the budget-constrained multiproduct problem. In the first set of examples we consider 3 products with independent demands. Thus we assume $\sigma_{i j}=d_{i j}=0$ for all $i \neq j, i=1,2,3, j=1,2,3$. The cost and demand parameters for the products are listed in Table 3 . The parameters are selected to facilitate exploring the effect of demand variability and the second-stage purchase cost. Hence we keep the salvage value, stockout cost, first-stage purchase cost, selling price and estimate of mean demand at time 1 identical across the three products. The optimal first-stage order quantities in the 
Table 4

Optimal first-stage order quantities and expected profit for the problem described in Table 3.

\begin{tabular}{llllll}
\hline$B$ & $Q_{11}$ & $Q_{12}$ & $Q_{13}$ & maxprofit & profit-sim \\
\hline 260 & 17.1 & 17.8 & 17.8 & 720.2 & 720.2 \\
280 & 18.5 & 18.9 & 18.6 & 761.2 & 761.2 \\
300 & 20.0 & 20.0 & 20.0 & 792.4 & 792.4 \\
320 & 20.3 & 19.9 & 20.7 & 814.5 & 815.7 \\
340 & 20.6 & 20.3 & 21.6 & 829.0 & 830.3 \\
360 & 20.8 & 20.7 & 22.3 & 836.5 & 837.9 \\
380 & 20.6 & 20.9 & 22.5 & 839.6 & 841.1 \\
400 & 20.5 & 20.9 & 22.5 & 840.8 & 842.3 \\
\hline
\end{tabular}

Table 5

Optimal order quantities and expected profit for the single-stage problem with parameters given in Table 3.

\begin{tabular}{lllll}
\hline$B$ & $Q_{11}$ & $Q_{12}$ & $Q_{13}$ & maxprofit \\
\hline 260 & 17.1 & 17.8 & 17.1 & 720.2 \\
280 & 18.5 & 18.9 & 18.6 & 761.2 \\
300 & 20.0 & 20.0 & 20.0 & 792.4 \\
320 & 21.5 & 21.1 & 21.5 & 813.2 \\
340 & 22.9 & 22.2 & 22.9 & 824.2 \\
360 & 24.1 & 23.0 & 24.1 & 826.7 \\
380 & 24.1 & 23.0 & 24.1 & 826.7 \\
400 & 24.1 & 23.0 & 24.1 & 826.7 \\
\hline
\end{tabular}

unconstrained-budget case are $Q_{11}=20.4, \quad Q_{12}=20.9$, and $Q_{13}=22.4$, resulting in a total expected profit of 842.6. As discussed in Section 4, the optimal solution for the unconstrained-budget case can be found by solving 3 single-product problems separately.

The optimal solutions with different levels of budget availability are displayed in Table 4 . The optimal expected profit at time 1 , maxprofit, increases as the available budget $B$ increases. The marginal benefit of additional budget gets smaller as the budget amount becomes larger, and the expected profit approaches the expected profit for the unconstrained-budget case. The expected profit calculated by simulating the random vector $\mu_{2}$ is denoted by profit-sim in Table 4. The small difference between maxprofit and profit-sim indicates that the error caused by discretizing the pdf of $\mu_{2}$ in evaluating (20) is not expected to be significant. Note that when the budget is restricted, the problem turns into a single-stage problem which is solved without taking into account the pdf of $\mu_{2}$.

Similar to the single-product case discussed in Section 7.1, we compare the optimal solution for the single-order problem (presented in Table 5) with that for the two-order case (Table 4). As noted previously, demand for product $i$ at time 1 is distributed as normal with mean $\mu_{1 i}$ and variance $\sigma_{i i}+d_{i i}$. The difference between the profits in Tables 4 and 5 shows a similar pattern to the difference between the profits in Tables 1 and 2 . The benefit from the demand forecast update is realized only when the budget is sufficiently large. When there is no financial constraint, the order sizes for all products in the single-stage problem exceed the initial order sizes in the two-stage problem.

We note that, since all products have the same cost parameters, the optimal solution to the single-stage problem when the budget constraint is active can be found using (see Erlebacher, 2000, Theorem 1):

$Q_{1 i}=\mu_{1 i}+\left(\sigma_{i i}+d_{i i}\right)^{0.5} \frac{B-\sum_{i=1}^{3} c_{1 i} \mu_{1 i}}{\sum_{i=1}^{3} c_{1 i}\left(\sigma_{i i}+d_{i i}\right)^{0.5}}, \quad i=1,2,3$.

According to (23), the impact of demand standard deviation on the order quantity of a product depends on the sign of
$B-\sum_{i=1}^{3} c_{1 i} \mu_{1 i}$. If $B<\sum_{i=1}^{3} c_{1 i} \mu_{1 i}$, a higher value for $\left(\sigma_{i i}+d_{i i}\right)^{0.5}$ implies a lower order size for product $i$. On the other hand, if $B>\sum_{i=1}^{3} c_{1 i} \mu_{1 i}$, a higher value for $\left(\sigma_{i i}+d_{i i}\right)^{0.5}$ implies a larger order size for product $i$. Notice that the effect of demand standard deviation on the order size is independent of the critical fractile value, which is determined by the underage and overage costs. We remark that in the traditional single-product newsboy problem with normally distributed demand, the optimal order size equals mean demand plus $k \times$ (standard deviation of demand), where the multiplier $k$ depends on the critical fractile. Hence, in the single-product problem, the qualitative effect of a change in demand standard deviation on the optimal order size depends on the sign of $k$, or equivalently, whether the order size is above or below the mean demand. From (23), we have

$\frac{\partial Q_{1 i}}{\partial B}=\frac{\left(\sigma_{i i}+d_{i i}\right)^{0.5}}{\sum_{i=1}^{3} c_{1 i}\left(\sigma_{i i}+d_{i i}\right)^{0.5}}>0$.

It follows from Eq. (24) that

$\frac{\partial Q_{1 i}}{\partial B}>\frac{\partial Q_{1 j}}{\partial B} \quad$ if $\quad\left(\sigma_{i i}+d_{i i}\right)^{0.5}>\left(\sigma_{j j}+d_{j j}\right)^{0.5}$.

Thus, Eq. (25) indicates that as the budget increases, the increase in the order size of the product with a higher demand standard deviation is more than that of the product with a lower demand standard deviation. This expression explains the changes in the order sizes in response to budget changes in Table 5 .

Products 1 and 2 have the same cost parameters. Demand for product 1 is more volatile than that for product 2 . With a limited budget, the retailer plays safe, and diverts the limited funds to the product that entails a lower demand risk (product 2 ). However, as the budget increases, this preference is reversed and product 2 is given a lower weight in the retailer's product portfolio. When the funds are abundant (non-binding), the retailer can make stocking decision of a product independently of other products; hence, given the critical fractile values for products in this example, more demand variability results in a larger order quantity.

The result that the product with more predictable demand is allocated a higher share of the binding budget is also observed in the two-stage problem of Table 4 . As the budget increases, product 1 becomes more attractive for investment, and it accounts for a larger share of the total expenditure. But, as different from the single-stage problem in Table 5 , the order size for product 1 does not exceed the order size for product 2 as the budget constraint is loosened. Thus having the option to issue a second order prevents a large increase in the initial order for product 1 . Referring to the results in Table 4, we also notice that high unit purchase cost for product 3 at time 2 urges the retailer to place a large order of product 3 at time 1 .

Note that under a tight budget, the two-stage problem essentially turns into a budget-constrained single-stage problem, and as Eq. (23) implies, a high demand variance leads to a smaller order size. Hence, the order size of the more risky product (product 1 ) is smaller than that of the less risky product (product 2 ) in Table 4 for low budget amounts.

As noted earlier, in the traditional newsboy problem, the qualitative impact of demand variability on the order size depends on whether the critical fractile is above or below 0.5 . To investigate how critical fractile may influence the ordering policy in our problem, we change some of the cost parameters used in Table 3, and display the new data in Table 6. We decrease the selling price significantly so that the critical fractile at time 1 is less than 0.5 for all three products. The optimal decisions under new parameters are shown in Table 7. The optimal firststage order quantities in the unconstrained-budget case are $Q_{11}=17.2, Q_{12}=18.2$, and $Q_{13}=18.5$, and the optimal expected profit is 150.0 . 
Table 6

Cost and demand parameters in the second numerical example of products with independent demands.

\begin{tabular}{lllllllll}
\hline Product $(i)$ & $\tau_{i}$ & $\pi_{i}$ & $c_{1 i}$ & $c_{2 i}$ & $p_{i}$ & $\mu_{1 i}$ & $\sigma_{i i}$ & $d_{i i}$ \\
\hline 1 & 1 & 0 & 5 & 6 & 8 & 20 & 2 & 20 \\
2 & 1 & 0 & 5 & 6 & 8 & 20 & 2 & 10 \\
3 & 1 & 0 & 5 & 7 & 8 & 20 & 2 & 20 \\
\hline
\end{tabular}

Table 7

Optimal first-stage order quantities and expected profit for the problem described in Table 6.

\begin{tabular}{llllll}
\hline$B$ & $Q_{11}$ & $Q_{12}$ & $Q_{13}$ & maxprofit & profit-sim \\
\hline 220 & 14.1 & 15.7 & 14.2 & 127.4 & 127.4 \\
240 & 15.6 & 16.8 & 15.6 & 135.5 & 135.5 \\
260 & 17.1 & 17.8 & 17.1 & 141.4 & 141.4 \\
280 & 17.4 & 17.9 & 17.5 & 145.2 & 145.4 \\
300 & 17.4 & 17.8 & 18.0 & 147.8 & 148.1 \\
320 & 17.2 & 18.2 & 18.5 & 149.1 & 149.4 \\
340 & 17.2 & 18.2 & 18.5 & 149.6 & 149.9 \\
\hline
\end{tabular}

\section{Table 8}

Cost and demand parameters in the numerical example of products with dependent demands $\left(\sigma_{12}=2, \sigma_{13}=-1, \sigma_{23}=-2, d_{12}=10, d_{13}=-5, d_{23}=-8\right)$.

\begin{tabular}{lllllllll}
\hline Product $(i)$ & $\tau_{i}$ & $\pi_{i}$ & $c_{1 i}$ & $c_{2 i}$ & $p_{i}$ & $\mu_{1 i}$ & $\sigma_{i i}$ & $d_{i i}$ \\
\hline 1 & 1 & 2 & 3 & 5 & 12 & 10 & 2 & 10 \\
2 & 1 & 2 & 3 & 5 & 12 & 15 & 3 & 15 \\
3 & 1 & 2 & 3 & 7 & 12 & 15 & 3 & 10 \\
\hline
\end{tabular}

The results in Table 7 are similar to that in Table 4 . When the budget is in the intermediate range (280-300), the order size for product 1 is greater than the order size for the unconstrainedbudget case (17.2). It appears that when the budget is large, in the high critical fractile case (Table 4), the difference between the order sizes of product 2 and product 1 is less than that in the low critical fractile case (Table 7). We can link this result to the impact of demand variance in the traditional newsboy problem. In the newsboy problem with normally distributed demand, order size is negatively related to demand variance when the critical fractile is below 0.5 . In our problem, the preference given to the product with less variable demand (product 2) over the product with more variable demand (product 1 ) seems to increase when the critical fractile is low. As in Table 4, the difference between maxprofit and profit-sim is not significant, indicating that (21) provides a good approximation for (20).

\subsection{Multi-product problem with dependent demands}

Finally we present examples for the case of products with correlated demands. The cost and demand distribution parameters for the first example are listed in Table 8 . There are three products, and demands for product 1 and product 2 are positively correlated. The coefficient of correlation between product 1 and product 3 as well as between product 2 and product 3 are both negative. By using the algorithm described in Section 6, we obtain the results shown in Table 9. The optimal solution for the unconstrained-budget problem is $Q_{11}=12.2, Q_{12}=17.7$, and $Q_{13}=18.3$, with an associated expected profit of 328.7.

The general patterns in Table 9 are similar to that in Tables 4 and 7. When the budget is in a certain interval, the order sizes for product 1 and product 2 exceed the order sizes for the unconstrainedbudget case. Because the demand for product 3 is less volatile than
Table 9

Optimal first-stage order quantities and expected profit for the problem described in Table 8.

\begin{tabular}{lrrrll}
\hline B & $Q_{11}$ & $Q_{12}$ & $Q_{13}$ & maxprofit & profit-sim \\
\hline 110 & 9.0 & 13.7 & 13.9 & 283.7 & 283.7 \\
120 & 10.0 & 15.0 & 15.0 & 301.3 & 301.3 \\
130 & 11.0 & 16.3 & 16.0 & 313.1 & 313.9 \\
140 & 12.0 & 17.5 & 17.1 & 320.7 & 321.6 \\
150 & 12.4 & 18.0 & 17.6 & 324.5 & 325.4 \\
160 & 12.5 & 18.0 & 17.9 & 326.3 & 327.3 \\
170 & 12.4 & 17.9 & 18.0 & 327.2 & 328.1 \\
180 & 12.3 & 17.8 & 18.1 & 327.6 & 328.6 \\
\hline
\end{tabular}

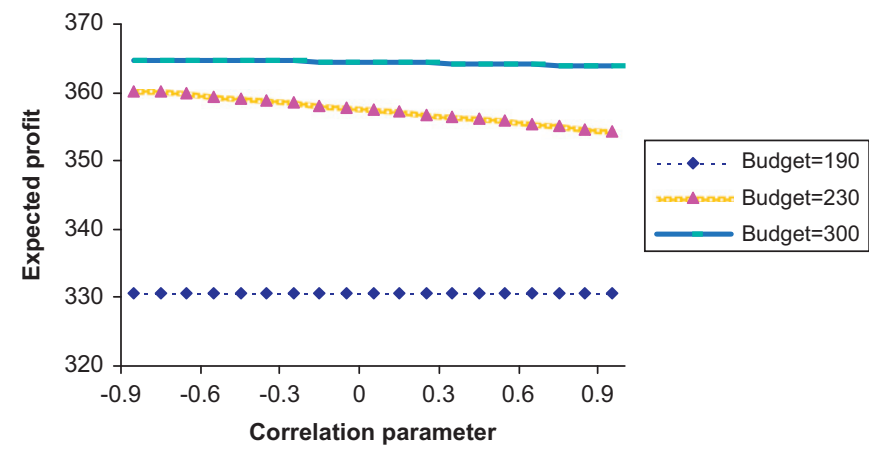

Fig. 4. Effect of correlation parameter $\rho$ on expected profit at different budget levels in the two-product problem.

demand for product 2, and also the purchase cost at time 2 of product 3 is higher, the initial order size for product 3 is higher than that for product 2 when the budget is limited. It is observed in Table 9 that when the budget is in the intermediate range, $Q_{12}$ slightly exceeds $Q_{13}$, which suggests that the combined effect of demand variability and correlations among product demands offset the effect of purchase cost when the budget is in this range.

To investigate the impact of positive versus negative correlation among product demands, we solved a two-product problem with varying degrees of correlation among demands. We used the parameters $\quad \tau_{1}=\tau_{2}=1, \quad \pi_{1}=\pi_{2}=2, \quad p_{1}=p_{2}=15, \quad c_{11}=c_{12}=5$, $c_{21}=c_{22}=7, \quad \mu_{11}=\mu_{12}=20, \quad \sigma_{11}=\sigma_{22}=2, \quad \sigma_{12}=\rho\left(\sigma_{11} \sigma_{22}\right)^{0.5}$, $d_{11}=d_{22}=20, d_{12}=\rho\left(d_{11} d_{22}\right)^{0.5}$. Thus by varying the parameter $\rho$, we can modify the nature of dependence between product demands. Positive (negative) values for $\rho$ indicate that high levels of demand for a product will be more likely to occur when the demand for the other product is high (low). We observed that the impact of correlation on expected profit depends on the budget amount. When the budget is very limited or very large, the parameter $\rho$ is observed to have a negligible impact on expected profit. Using a budget of $B \in\{190,230,300\}$, we solved the problem with $\rho$ values between -0.9 and 0.9 with a step size of 0.1 . When the budget is very tight $(B=190)$, all funds are spent at time 1 so the new market information is not important for determining the optimal policy. When the budget is unrestricted $(B=300)$, the retailer is less pressured to consider the possibility of monetary constraint at time 2. Only when the budget is in the midrange $(B=230)$ that allows to carry a limited amount of funds to time 2 , the correlation parameter $\rho$ has some influence on expected profit.

The optimal expected profit and optimal initial orders as a function of $\rho$ are plotted in Figs. 4 and 5, respectively. The results for $B=230$ indicate that as $\rho$ increases from -0.9 to 0.9 , the optimal initial order quantities for products increase while the optimal expected profit decreases. The optimal initial orders when $\rho$ is negative are less than that when $\rho$ is zero; when 


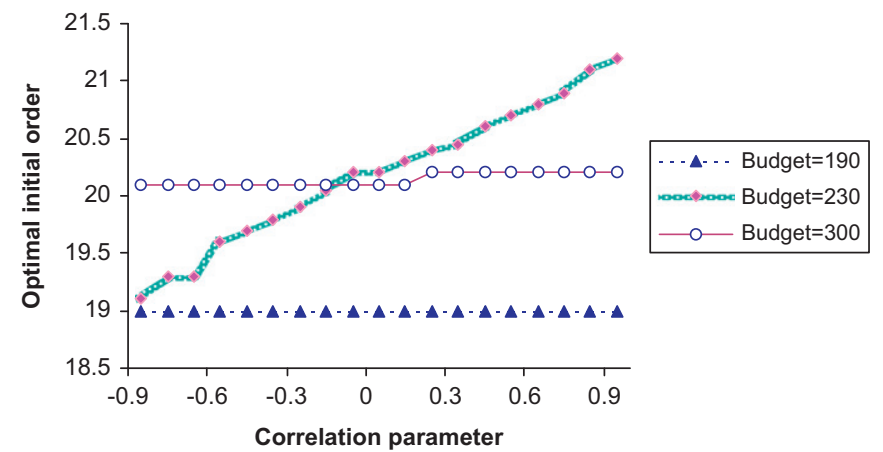

Fig. 5. Effect of correlation parameter $\rho$ on optimal initial order at different budget levels in the two-product problem.

product demands are negatively correlated, the retailer reserves some funds for stage 2 . At time 2 , this reserved money can be used to purchase more of the product that has a higher demand signal after the new market information is gathered. Interestingly, when the budget is 230 , positive correlation between demands may lead to an initial order exceeding that when the budget is 300. Thus positive correlation between product demands causes the retailer to order more at time 1 , which is similar to how a budgetary constraint may lead to an increase in the initial order quantity.

We find that correlation between product demands has a minor impact on expected profit as the difference between the expected profits when $\rho=-0.9$ and when $\rho=0.9$ is less than $2 \%$ in Fig. 4. We observed similar results in other numerical examples with different cost and demand data.

\section{Conclusion}

We have studied an extension of the budget-constrained multi-product newsvendor problem in which two separate orders can be placed at two different times, and a Bayesian updating of demand forecast is made at the time of the second order. A multivariate normal demand distribution has been assumed. A dynamic programming algorithm is used to determine the optimal allocation of the budget among competing products. The algorithm requires solving a series of constrained multiproduct newsvendor problems with given initial inventories. The problem can also be regarded as an extension of the two-stage ordering problem of a single product in a quick response system to the setting of multiple products. In the budget-constrained single-product case, we have derived the optimality equation that the first-stage order quantity should satisfy.

We have shown that in the single-product problem, a limited budget may cause the initial order size to be greater than the order size under unlimited budget, and high supply cost for an item at the second stage leads to an increase in the quantity of that item ordered at the first stage. Similar patterns are observed in the numerical examples involving multiple products. The numerical examples illustrate the increase in the expected profit resulting from placing two orders instead of the traditional single order. As the purchasing budget is reduced, cost savings due to demand forecast revision decrease.

The allocation of the budget among the multiple products with the same cost structure was studied. The results indicate that the amount of funds available has an important effect on the relative share of products in the portfolio. In the unlimited budget case with independent product demands, products with high demand volatility make up a large portion of the total procurement expenditure. However, when the budget is scarce, products with less volatile demand are given preference in the assortment.

Future research directions include developing effective heuristics for finding an approximately optimal solution, investigating the case of price-sensitive demand, and incorporating risk measures such as conditional value-at-risk (CVaR).

\section{Acknowledgment}

The author gratefully acknowledges the comments by a referee, which helped to improve the presentation significantly.

\section{Appendix A}

Derivation of Eqs. (1) and (2):

If $K$ is a $p$-variate normal random variable with mean $\mu_{2}$ and covariance matrix $D_{2}$, the density is given by

$\gamma\left(K \mid \mu_{2}, D_{2}\right)=\frac{1}{(2 \pi)^{0.5 p} \sqrt{\operatorname{det}\left(D_{2}\right)}} \exp \left[-0.5\left(K-\mu_{2}\right) D_{2}^{-1}\left(K-\mu_{2}\right)^{T}\right]$.

Since

$\left(K-\mu_{2}\right) D_{2}^{-1}\left(K-\mu_{2}\right)^{T}=K D_{2}^{-1} K^{T}-K D_{2}^{-1} \mu_{2}^{T}-\mu_{2} D_{2}^{-1} K^{T}+\mu_{2} D_{2}^{-1} \mu_{2}^{T}$,

and

$\mu_{2} D_{2}^{-1} K^{T}=\left(\mu_{2} D_{2}^{-1} K^{T}\right)^{T}=K\left(D_{2}^{-1}\right)^{T} \mu_{2}^{T}=K D_{2}^{-1} \mu_{2}^{T}$,

the density can be written as

$\gamma\left(K \mid \mu_{2}, D_{2}\right) \propto \exp \left[K D_{2}^{-1} K^{T}-2 K D_{2}^{-1} \mu_{2}^{T}\right]$.

The terms not involving $K$ are omitted on the right side of (A1). Let $\alpha(M)$ be the prior density of $M$. It is assumed that

$\alpha(M)=\frac{1}{(2 \pi)^{0.5 p} \sqrt{\operatorname{det}(D)}} \exp \left[-0.5\left(M-\mu_{1}\right) D^{-1}\left(M-\mu_{1}\right)^{T}\right]$.

The likelihood function $L(X \mid M)$ is

$L(X \mid M)=\frac{1}{(2 \pi)^{0.5 p} \sqrt{\operatorname{det}\left(\Sigma_{1}\right)}} \exp \left[-0.5(X-M) \Sigma_{1}^{-1}(X-M)^{T}\right]$.

By Bayesian approach, the posterior density of $M, f(M \mid X)$ is proportional to the likelihood function multiplied by the prior density of $M$ :

$f(M \mid X) \propto L(X \mid M) \alpha(M)$.

Thus, we have

$f(M \mid X) \propto \exp \left[(M-X) \Sigma_{1}^{-1}(M-X)^{T}+\left(M-\mu_{1}\right) D^{-1}\left(M-\mu_{1}\right)^{T}\right]$.

Rewriting the term on the right side of (A2),

$$
\begin{aligned}
& (M-X) \Sigma_{1}^{-1}(M-X)^{T}+\left(M-\mu_{1}\right) D^{-1}\left(M-\mu_{1}\right)^{T}=M \Sigma_{1}^{-1} M^{T}-M \Sigma_{1}^{-1} X^{T} \\
& \quad-X \Sigma_{1}^{-1} M^{T}+X \Sigma_{1}^{-1} X^{T}+M D^{-1} M^{T}-M D^{-1} \mu_{1}^{T}-\mu_{1} D^{-1} M^{T}+\mu_{1} D^{-1} \mu_{1}^{T}
\end{aligned}
$$

Collecting the terms,

$$
\begin{gathered}
(M-X) \Sigma_{1}^{-1}(M-X)^{T}+\left(M-\mu_{1}\right) D^{-1}\left(M-\mu_{1}\right)^{T}=M\left(D^{-1}+\Sigma_{1}^{-1}\right) M^{T} \\
-2 M\left(\Sigma_{1}^{-1} X^{T}+D^{-1} \mu_{1}^{T}\right)+h\left(X, \mu_{1}, \Sigma_{1}, D\right)
\end{gathered}
$$

The terms that do not depend on $M$ are shown by $h\left(X, \mu_{1}, \Sigma_{1}, D\right)$ in (A4). Thus, using (A2) and (A4),

$f(M \mid X) \propto \exp \left[M\left(D^{-1}+\Sigma_{1}^{-1}\right) M^{T}-2 M\left(\Sigma_{1}^{-1} X^{T}+D^{-1} \mu_{1}^{T}\right)\right]$.

To obtain a proper probability density function, the right side of (A5) should be multiplied by a normalization constant that does not depend on $M$. Comparing (A1) and (A5), we observe $D_{2}=\left(D^{-1}+\Sigma_{1}^{-1}\right)^{-1}$ and $D_{2}^{-1} \mu_{2}^{T}=\Sigma_{1}^{-1} X^{T}+D^{-1} \mu_{1}^{T}$. It follows that the posterior density of the parameter vector $M, f(M \mid X)$ is 
$p$-variate normal with mean $\mu_{2}=\left[D_{2}\left(\Sigma_{1}^{-1} X^{T}+D^{-1} \mu_{1}^{T}\right)\right]^{T}$ and covariance matrix $D_{2}=\left(D^{-1}+\Sigma_{1}^{-1}\right)^{-1}$.

Proof of Proposition 1. Because $E P_{1 i}\left(Q_{1 i}\right)$ depends only on $Q_{1 i}$ and not on $Q_{1 j}, j \neq i$, it is sufficient to show concavity of $E P_{1 i}\left(Q_{1 i}\right)$ in $Q_{1 \text { i. Using (10), we obtain }}$

$$
\frac{\partial^{2} E P_{1 i}\left(Q_{1 i}\right)}{\partial Q_{1 i}^{2}}=-\frac{\left(p_{i}+\pi_{i}-\tau_{i}\right)}{a_{i i}^{0.5}} \int_{-\infty}^{\kappa_{i}} \varphi\left(\frac{Q_{1 i}-\mu_{1 i}-\gamma_{i} v_{i i}^{0.5}}{a_{i i}^{0.5}}\right) \varphi\left(\gamma_{i}\right) d \gamma_{i} \leq 0 .
$$

Hence, the expected profit associated with product $i, E P_{1 i}\left(Q_{1 i}\right)$ is concave in $Q_{1 i}$. We note that in the budget-unconstrained multiproduct problem, the order quantity $Q_{1 i}$ for a product indirectly depends on the demand distribution characteristics of other products because, by (3), the variance of the distribution of $\mu_{2 i}$, $v_{i i}$ is a function of the elements of the covariance matrices $\Sigma_{1}$ and $D$. Thus the optimal $Q_{1 i}$ is not totally independent of the demand distribution parameters of other products.

Proof of Proposition 2. We first show that the expected profit function $E P\left(Q_{1}\right)$ given by (15) is continuous. Using (12) and (13), it can be verified that

$$
\begin{gathered}
\left.T_{1}\left(Q_{1}, \mu_{2}\right)\right|_{\mu_{2}=Q_{1}-\sigma_{x} \Phi^{-1}(s)}=\left.T_{2}\left(Q_{1}, \mu_{2}\right)\right|_{\mu_{2}=Q_{1}-\sigma_{x} \Phi^{-1}(s)} \\
=p Q_{1}+(\tau-p) \sigma_{x} \Phi^{-1}(s)-(p+\pi-\tau) \sigma_{x} \Psi\left(\Phi^{-1}(s)\right)
\end{gathered}
$$

Similarly, it can be checked that

$$
\begin{aligned}
& \left.T_{2}\left(Q_{1}, \mu_{2}\right)\right|_{\mu_{2}=Q_{1}-\sigma_{x} \Phi^{-1}(s)+\frac{B-c_{1} Q_{1}}{c_{2}}}=\left.T_{3}\left(Q_{1}, \mu_{2}\right)\right|_{\mu_{2}=Q_{1}-\sigma_{x} \Phi^{-1}(s)+\frac{B-c_{1} Q_{1}}{c_{2}}} \\
& =p\left(Q_{1}+\frac{B-c_{1} Q_{1}}{c_{2}}\right)+(\tau-p) \sigma_{x} \Phi^{-1}(s) \\
& -(p+\pi-\tau) \sigma_{x} \Psi\left(\Phi^{-1}(s)\right)-\left(B-c_{1} Q_{1}\right) .
\end{aligned}
$$

Thus $E P\left(Q_{1}\right)$ is continuous as $\mu_{2}$ changes. From (12)-(14), observe that

$$
\begin{aligned}
& \frac{\partial T_{1}\left(Q_{1}, \mu_{2}\right)}{\partial Q_{1}}=\tau+(p+\pi-\tau) \Phi_{c}\left(\frac{Q_{1}-\mu_{2}}{\sigma_{x}}\right), \\
& \frac{\partial T_{2}\left(Q_{1}, \mu_{2}\right)}{\partial Q_{1}}=c_{2}, \\
& \frac{\partial T_{3}\left(Q_{1}, \mu_{2}\right)}{\partial Q_{1}}=\left(1-\frac{c_{1}}{c_{2}}\right)\left[\tau+(p+\pi-\tau) \Phi_{c}\left(\frac{W-\mu_{2}}{\sigma_{x}}\right)\right]+c_{1},
\end{aligned}
$$

where $\Phi_{c}(\cdot)$ is the complementary cdf for the standard normal distribution. By using (A7)-(A9) and (15), the first derivative of $E P\left(Q_{1}\right)$ with respect to $Q_{1}$ is

$$
\begin{aligned}
& \frac{\partial E P\left(Q_{1}\right)}{\partial Q_{1}}=\int_{-\infty}^{Q_{1}-\sigma_{x} \Phi^{-1}(s)}\left[\tau+(p+\pi-\tau) \Phi_{c}\left(\frac{Q_{1}-\mu_{2}}{\sigma_{x}}\right)\right] g\left(\mu_{2}\right) d \mu_{2} \\
& \quad+c_{2}\left[\Phi\left(\frac{W-\sigma_{x} \Phi^{-1}(s)-\mu_{1}}{\sigma}\right)-\Phi\left(\frac{Q_{1}-\sigma_{x} \Phi^{-1}(s)-\mu_{1}}{\sigma}\right)\right] \\
& +\int_{Q_{1}-\sigma_{x} \Phi^{-1}(s)+\left(\left(B-c_{1} Q_{1}\right) / c_{2}\right)}^{\infty}\left\{c_{1}+\left(1-\frac{c_{1}}{c_{2}}\right)\left[\tau+(p+\pi-\tau) \Phi_{c}\left(\frac{W-\mu_{2}}{\sigma_{x}}\right)\right]\right\} \\
& g\left(\mu_{2}\right) d \mu_{2}-c_{1},
\end{aligned}
$$

where $\sigma$ is the standard deviation of the distribution of $\mu_{2}$. In order to show that the second derivative of $E P\left(Q_{1}\right)$ is negative, we will decompose the right side of (A10) into three parts as follows:

$\frac{\partial E P\left(Q_{1}\right)}{\partial Q_{1}}=Z_{1}+Z_{2}+Z_{3}$.

Define $Z_{1}$ as

$$
\begin{aligned}
Z_{1}= & \int_{-\infty}^{Q_{1}-\sigma_{x} \Phi^{-1}(s)}\left[\tau+(p+\pi-\tau) \Phi_{c}\left(\frac{Q_{1}-\mu_{2}}{\sigma_{x}}\right)\right] g\left(\mu_{2}\right) d \mu_{2} \\
& -c_{2} \Phi\left(\frac{Q_{1}-\sigma_{x} \Phi^{-1}(s)-\mu_{1}}{\sigma}\right) .
\end{aligned}
$$

From (A11)

$\frac{\partial Z_{1}}{\partial Q_{1}}=-(p+\pi-\tau) \int_{-\infty}^{Q_{1}-\sigma_{x} \Phi^{-1}(s)} \frac{1}{\sigma_{x}} \varphi\left(\frac{Q_{1}-\mu_{2}}{\sigma_{x}}\right) g\left(\mu_{2}\right) d \mu_{2}<0$.

Define $Z_{2}$ as

$Z_{2}=\int_{Q_{1}-\sigma_{x} \Phi^{-1}(s)+\left(\left(B-c_{1} Q_{1}\right) / c_{2}\right)}^{\infty}\left\{c_{1}+\left(1-\frac{c_{1}}{c_{2}}\right)\left[\tau+(p+\pi-\tau) \Phi_{c}\left(\frac{W-\mu_{2}}{\sigma_{x}}\right)\right]\right\}$
$\quad g\left(\mu_{2}\right) d \mu_{2}-c_{1}$.

We have

$$
\begin{gathered}
\frac{\partial Z_{2}}{\partial Q_{1}}=\frac{-1}{\sigma}\left(1-\frac{c_{1}}{c_{2}}\right)\left[(p+\pi)\left(1-\frac{c_{1}}{c_{2}}\right)+c_{1}\right] \varphi\left(\frac{W-\Phi^{-1}(s) \sigma_{x}-\mu_{1}}{\sigma}\right) \\
-(p+\pi-\tau) \int_{Q_{1}-\sigma_{x} \Phi^{-1}(s)+\left(\left(B-c_{1} Q_{1}\right) / c_{2}\right)}^{\infty} \frac{1}{\sigma_{x}}\left(1-\frac{c_{1}}{c_{2}}\right) \varphi\left(\frac{W-\mu_{2}}{\sigma_{x}}\right) g\left(\mu_{2}\right) d \mu_{2} \\
+\frac{1}{\sigma}\left(1-\frac{c_{1}}{c_{2}}\right)^{2}\left(p+\pi-c_{2}\right) \varphi\left(\frac{W-\Phi^{-1}(s) \sigma_{x}-\mu_{1}}{\sigma}\right)
\end{gathered}
$$

Now define $Z_{3}$ as

$Z_{3}=c_{2} \Phi\left(\frac{W-\sigma_{x} \Phi^{-1}(s)-\mu_{1}}{\sigma}\right)$

Differentiating (A15), we obtain

$\frac{\partial Z_{3}}{\partial Q_{1}}=\frac{1}{\sigma}\left(c_{2}-c_{1}\right) \varphi\left(\frac{W-\Phi^{-1}(s) \sigma_{x}-\mu_{1}}{\sigma}\right)$.

From (A14) and (A16) we obtain

$$
\begin{aligned}
& \frac{\partial Z_{2}}{\partial Q_{1}}+\frac{\partial Z_{3}}{\partial Q_{1}}=-(p+\pi-\tau) \int_{Q_{1}-\sigma_{x} \Phi^{-1}(s)+\left(\left(B-c_{1} Q_{1}\right) / c_{2}\right)}^{\infty} \frac{1}{\sigma_{x}}\left(1-\frac{c_{1}}{c_{2}}\right) \varphi \\
& \quad\left(\frac{W-\mu_{2}}{\sigma_{x}}\right) g\left(\mu_{2}\right) d \mu_{2}<0 .
\end{aligned}
$$

Finally, (A12) and (A17) imply that

$$
\frac{\partial^{2} E P\left(Q_{1}\right)}{\partial Q_{1}^{2}}<0
$$

Proof of Proposition 3. We first show that for a fixed $Q_{1}, E P$ is non-decreasing concave in $B$. From (15), we have

$\frac{\partial E P}{\partial B}=\int_{Q_{1}-\sigma_{x} \Phi^{-1}(s)+\left(\left(B-c_{1} Q_{1}\right) / c_{2}\right)}^{\infty} \frac{\partial T_{3}\left(Q_{1}, \mu_{2}\right)}{\partial B} g\left(\mu_{2}\right) d \mu_{2}$.

Differentiating (14), we obtain

$\frac{\partial T_{3}\left(Q_{1}, \mu_{2}\right)}{\partial B}=\frac{p+\pi-c_{2}}{c_{2}}-\frac{p+\pi-\tau}{c_{2}} \Phi\left[\frac{Q_{1}\left(1-\left(c_{1} / c_{2}\right)\right)+\left(B / c_{2}\right)-\mu_{2}}{\sigma_{x}}\right]$.

When the budget is constraining, we have

$\Phi\left[\frac{Q_{1}\left(1-\left(c_{1} / c_{2}\right)\right)+\left(B / c_{2}\right)-\mu_{2}}{\sigma_{x}}\right] \leq s=\frac{p+\pi-c_{2}}{p+\pi-\tau}$.

(A19) and (A20) imply that $\left(\left(\partial T_{3}\left(Q_{1}, \mu_{2}\right)\right) / \partial B\right) \geq 0$, and consequently

$\frac{\partial E P}{\partial B}=\int_{Q_{1}-\sigma_{x} \Phi^{-1}(s)+\left(\left(B-c_{1} Q_{1}\right) / c_{2}\right)}^{\infty} \frac{\partial T_{3}\left(Q_{1}, \mu_{2}\right)}{\partial B} g\left(\mu_{2}\right) d \mu_{2} \geq 0$

Using (A18),

$\frac{\partial^{2} E P}{\partial B^{2}}=\int_{Q_{1}-\sigma_{x} \Phi^{-1}(s)+\frac{B-c_{1} Q_{1}}{c_{2}}}^{\infty} \frac{\partial^{2} T_{3}\left(Q_{1}, \mu_{2}\right)}{\partial B^{2}} g\left(\mu_{2}\right) d \mu_{2}$

Taking the derivative of (A19), we have

$\frac{\partial^{2} T_{3}\left(Q_{1}, \mu_{2}\right)}{\partial B^{2}}=-\frac{p+\pi-\tau}{c_{2}^{2} \sigma_{x}} \varphi\left[\frac{Q_{1}\left(1-\left(c_{1} / c_{2}\right)\right)+\left(B / c_{2}\right)-\mu_{2}}{\sigma_{x}}\right] \leq 0$. 
Combining (A22) and (A23), we obtain

$\frac{\partial^{2} E P}{\partial B^{2}}=\int_{Q_{1}-\sigma_{x} \Phi^{-1}(s)+\left(\left(B-c_{1} Q_{1}\right) / c_{2}\right)}^{\infty} \frac{\partial^{2} T_{3}\left(Q_{1}, \mu_{2}\right)}{\partial B^{2}} g\left(\mu_{2}\right) d \mu_{2} \leq 0$.

The additional budget can be spent for increasing $Q_{1}$ and/or the purchase amount at time 2 . When $Q_{1}$ is fixed, an increase in budget can be used to purchase more units at time 2. Eqs. (A21) and (A24) imply that for a given $Q_{1}$, the expected profit increases at a decreasing rate as the budget increases. We have a linear purchase cost, and by Proposition 2, the expected profit is concave in $Q_{1}$. Thus, because the expected profit increases at a decreasing rate regardless of how the additional budget is spent, it follows that expected profit is non-decreasing concave in the budget amount $B$.

Proof of Proposition 4. The optimal initial order in the budgetunconstrained single-product problem $Q_{1}^{u}$ satisfies the following first-order optimality condition, which is found by setting Eq. (10) to zero:

$(p+\pi-\tau) \int_{-\infty}^{Q_{1}^{u}-\sigma_{x} \Phi^{-1}(s)} \Phi\left(\frac{Q_{1}^{u}-\mu_{2}}{\sigma_{x}}\right) g\left(\mu_{2}\right) d \mu_{2}-\left(p+\pi-c_{2}\right) \Phi(\zeta)=c_{2}-c_{1}$,

where $\zeta=\left(Q_{1}^{u}-\sigma_{x} \Phi^{-1}(s)-\mu_{1}\right) / \sigma$. From (A10), the first-order condition in the budget-constrained single-product problem is

$$
\begin{aligned}
& (p+\pi-\tau) \int_{-\infty}^{Q_{1}-\sigma_{x} \Phi^{-1}(s)} \Phi\left(\frac{Q_{1}-\mu_{2}}{\sigma_{x}}\right) g\left(\mu_{2}\right) d \mu_{2} \\
& -\left(p+\pi-c_{2}\right) \Phi\left(\frac{Q_{1}-\sigma_{x} \Phi^{-1}(s)-\mu_{1}}{\sigma}\right) \\
& \quad=c_{1} \Phi_{c}\left(\frac{W-\sigma_{x} \Phi^{-1}(s)-\mu_{1}}{\sigma}\right)+\int_{Q_{1}-\sigma_{x} \Phi^{-1}(s)+\frac{B-c_{1} Q_{1}}{c_{2}}}^{\infty} \\
& \left(1-\frac{c_{1}}{c_{2}}\right)\left[\tau+(p+\pi-\tau) \Phi_{c}\left(\frac{W-\mu_{2}}{\sigma_{x}}\right)\right] \\
& \quad \times g\left(\mu_{2}\right) d \mu_{2}+c_{2} \Phi\left(\frac{W-\sigma_{x} \Phi^{-1}(s)-\mu_{1}}{\sigma}\right)-c_{1}
\end{aligned}
$$

Recall that $W=Q_{1}\left(1-\left(c_{1} / c_{2}\right)\right)+\left(B / c_{2}\right)$. Observe that,

$$
\begin{gathered}
\int_{Q_{1}-\sigma_{x} \Phi^{-1}(s)+\left(\left(B-c_{1} Q_{1}\right) / c_{2}\right)}^{\infty}\left(1-\frac{c_{1}}{c_{2}}\right)\left[\tau+(p+\pi-\tau) \Phi_{c}\left(\frac{W-\mu_{2}}{\sigma_{x}}\right)\right] g\left(\mu_{2}\right) d \mu_{2} \\
\geq\left(c_{2}-c_{1}\right) \Phi_{c}\left(\frac{W-\sigma_{x} \Phi^{-1}(s)-\mu_{1}}{\sigma}\right)
\end{gathered}
$$

Eq. (A27) implies that

$$
\begin{aligned}
& c_{1} \Phi_{c}\left(\frac{W-\sigma_{x} \Phi^{-1}(s)-\mu_{1}}{\sigma}\right)+\int_{Q_{1}-\sigma_{x} \Phi^{-1}(s)+\frac{B-c_{1} Q_{1}}{c_{2}}}^{\infty} \\
& \left(1-\frac{c_{1}}{c_{2}}\right)\left[\tau+(p+\pi-\tau) \Phi_{c}\left(\frac{W-\mu_{2}}{\sigma_{x}}\right)\right] g\left(\mu_{2}\right) d \mu_{2} \\
& +c_{2} \Phi\left(\frac{W-\sigma_{x} \Phi^{-1}(s)-\mu_{1}}{\sigma}\right) \geq c_{2} .
\end{aligned}
$$

Define the left side of $(\mathrm{A} 26)$ as $H\left(Q_{1}\right)$, i.e.,

$$
\begin{gathered}
H\left(Q_{1}\right)=(p+\pi-\tau) \int_{-\infty}^{Q_{1}-\sigma_{x} \Phi^{-1}(s)} \Phi\left(\frac{Q_{1}-\mu_{2}}{\sigma_{x}}\right) g\left(\mu_{2}\right) d \mu_{2} \\
-\left(p+\pi-c_{2}\right) \Phi\left(\frac{Q_{1}-\sigma_{x} \Phi^{-1}(s)-\mu_{1}}{\sigma}\right) .
\end{gathered}
$$

Differentiating (A29),

$$
\frac{\partial H\left(Q_{1}\right)}{\partial Q_{1}}=\frac{p+\pi-\tau}{\sigma_{x}} \int_{-\infty}^{Q_{1}-\sigma_{x} \Phi^{-1}(s)} \varphi\left(\frac{Q_{1}-\mu_{2}}{\sigma_{x}}\right) g\left(\mu_{2}\right) d \mu_{2} \geq 0 .
$$

Thus, $H\left(Q_{1}\right)$ is increasing in $Q_{1}$. By (A28), the right side of (A26) exceeds the right side of (A25). Comparing (A25) and (A26), and considering (A30), it follows that, when $B>c_{1} Q_{1}^{u}$, the optimal $Q_{1}$ satisfying (A26) should be greater than or equal to $Q_{1}^{u}$.

Proof of Proposition 5. To prove the result, we show that the expected profit function $E P\left(Q_{1}\right)$ is supermodular in $\left(Q_{1}, c_{2}\right)$. Supermodularity follows when the mixed partial derivative $\partial^{2} E P / \partial Q_{1} \partial c_{2}$ is nonnegative (Topkis, 1979). From (A10), we obtain

$$
\begin{aligned}
& \frac{\partial^{2} E P\left(Q_{1}\right)}{\partial Q_{1} \partial c_{2}}=\Phi\left(\frac{W-\sigma_{x} \Phi^{-1}(s)-\mu_{1}}{\sigma}\right)-\Phi\left(\frac{Q_{1}-\sigma_{x} \Phi^{-1}(s)-\mu_{1}}{\sigma}\right) \\
& \quad+\int_{W-\sigma_{x} \Phi^{-1}(s)}^{\infty} \frac{\partial}{\partial c_{2}}\left(\frac{c_{2}-c_{1}}{c_{2}}\right)\left[p+\pi-(p+\pi-\tau) \Phi\left(\frac{W-\mu_{2}}{\sigma_{x}}\right)\right] g\left(\mu_{2}\right) d \mu_{2} \\
& \quad+\int_{W-\sigma_{x} \Phi^{-1}(s)}^{\infty}\left(\frac{c_{2}-c_{1}}{c_{2}}\right)\left[-\frac{(p+\pi-\tau)}{\sigma_{x}} \frac{\partial W}{\partial c_{2}} \varphi\left(\frac{W-\mu_{2}}{\sigma_{x}}\right)\right] g\left(\mu_{2}\right) d \mu_{2} .
\end{aligned}
$$

Observe that

$\frac{\partial}{\partial c_{2}}\left(\frac{c_{2}-c_{1}}{c_{2}}\right)>0, \quad \frac{\partial W}{\partial c_{2}}<0$.

Since $W \geq Q_{1}$, the sum of the first two terms on the right-side of (A31) is nonnegative. By (A32), the third and fourth terms on the right-side of $(\mathrm{A} 31)$ are both positive. Hence it follows that $\left(\left(\partial^{2} E P\right) /\right.$ $\left.\left(\partial Q_{1} \partial c_{2}\right)\right) \geq 0$.

Proof of Proposition 6. It can be shown that $B_{2}\left(Q_{1}, Q_{2}, \mu_{2}\right)$ defined in (16) is jointly concave in $\left(Q_{11}, Q_{12}, \ldots, Q_{1 p}, Q_{21}, Q_{22}, \ldots, Q_{2 p}\right)$. Since the constraint is linear in $\left(Q_{1}, Q_{2}\right),(16)$ is a convex program. Then by convexity preservation theorem (e.g., Proposition B-4, Heyman and Sobel, 1984) and the fact that $\sum_{i=1}^{p} c_{1 i} Q_{1 i}$ is linear in $Q_{1 i}$, it follows that $E P\left(Q_{1}\right)$ defined in (18) is jointly concave in $Q_{1 i}, i=1$, $\ldots, p$.

\section{References}

Abdel-Malek, L.L., Areeratchakul, N., 2007. A quadratic programming approach to the multi-product newsvendor problem with side constraints. European Journal of Operational Research 176, 1607-1619.

Abdel-Malek, L.L., Montanari, R., 2005. On the multi-product newsboy problem with two constraints. Computers and Operations Research 32, 2095-2116.

Abdel-Malek, L., Montanari, R., Meneghetti, D., 2008. The capacitated newsboy problem with random yield: the Gardener Problem. International Journal of Production Economics 115, 113-127.

Abdel-Malek, L., Montanari, R., Morales, L.C., 2004. Exact, approximate, and generic iterative models for the multi-product newsboy problem with budget constraint. International Journal of Production Economics 91, 189-198.

Bhatnagar, R., Mehta, P., Teo, C.C., 2011. Coordination of planning and scheduling decisions in global supply chains with dual supply modes. International Journal of Production Economics 131, 473-482.

Bitran, G.R., Haas, E.A., Matsuo, H., 1986. Production planning of style goods with high setup costs and forecast revisions. Operations Research 34, 226-236.

Caro, F., Martinez-de-Albeniz, V., 2010. The impact of quick response in inventorybased competition. Manufacturing \& Service Operations Management 12, 409-429.

Chen, L.H., Chen, Y.C., 2010. A multiple-item budget-constraint newsboy problem with a reservation policy. Omega-International Journal of Management Science 38, 431-439.

Cheng, T.C.Edwin, Choi, Tsan-Ming (Eds.), 2010. Innovative Quick Response Programs in Logistics and Supply Chain Management. Springer, Heidelberg.

Choi, T.M., Chow, P.S., 2008. Mean-variance analysis of quick response program. International Journal of Production Economics 114, 456-475.

Choi, T.M., Li, D., Yan, H., 2003. Optimal two-stage ordering policy with Bayesian information updating. Journal of the Operational Research Society 54, 846-859.

Choi, T.M., Li, D., Yan, H., 2006. Quick response policy with Bayesian information updates. European Journal of Operational Research 170, 788-808.

Choi, T.M., Sethi, S., 2010. Innovative quick response programs: a review. International Journal of Production Economics 127, 1-12.

Chung, C.S., Flynn, J., Kirca, O., 2008. A multi-item newsvendor problem with preseason production and capacitated reactive production. European Journal of Operational Research 188, 775-792.

Donohue, K.L., 2000. Efficient supply contracts for fashion goods with forecast updating and two production modes. Management Science 46, 1397-1411. 
Erlebacher, S.J., 2000. Optimal and heuristic solutions for the multi-item newsvendor problem with a single capacity constraint. Production and Operations Management 9, 303-318.

Fernandes, N.O., Carmo-Silva, S., 2006. Generic POLCA-a production and materials flow control mechanism for quick response manufacturing. International Journal of Production Economics 104, 74-84.

Fisher, M., Raman, A., 1996. Reducing the cost of demand uncertainty through accurate response to early sales. Operations Research 44, 87-99.

Gurnani, H., Tang, C.S., 1999. Optimal ordering decisions with uncertain cost and demand forecast updating. Management Science 45, 1456-1462.

Hadley, H.G., Whitin, T.M., 1963. Analysis of Inventory Systems. Prentice-Hall, New Jersey.

Heyman, D.P., Sobel, M.J., 1984. Stochastic Models in Operations Research, vol. 2. McGraw-Hill, New York.

Huang, H., Sethi, S.P., Yan, H., 2005. Purchase contract management with demand forecast updates. IIE Transactions 37, 775-785.

Iyer, A.V., Bergen, M.E., 1997. Quick response in manufacturer-retailer channels. Management Science 43, 559-570.

Klosterhalfen, S., Kiesmuller, G., Minner, S., 2011. A comparison of the constantorder and dual-index policy for dual sourcing. International Journal of Production Economics 133, 302-311.

Lau, H.S., Lau, A.H.L., 1996. The newsstand problem: a capacitated multipleproduct single-period inventory problem. European Journal of Operational Research 94, 29-42.

Li, J., Chand, S., Dada, M., Mehta, S., 2009. Managing inventory over a short season: models with two procurement opportunities. Manufacturing \& Service Operations Management 11, 174-184.

Matsuo, H., 1990. A stochastic sequencing problem for style goods with forecast revisions and hierarchical structure. Management Science 36, 332-347.

Miltenburg, J., Pong, H.C., 2007a. Order quantities for style goods with two order opportunities and Bayesian updating of demand, Part I: no capacity constraints. International Journal of Production Research 45, 1643-1663.

Miltenburg, J., Pong, H.C., 2007b. Order quantities for style goods with two order opportunities and Bayesian updating of demand, Part II: capacity constraints. International Journal of Production Research 45, 1707-1723.

Moon, I., Silver, E.A., 2000. The multi-item newsvendor problem with a budget constraint and fixed ordering costs. Journal of the Operational Research Society $51,602-608$

Morey, R.C., Sweeney, D.J., 1984. A budget holdback policy for multi-item procurement processes. Management Science 30, 604-617.

Murray, G.R., Silver, E.A., 1966. A Bayesian analysis of the style goods inventory problem. Management Science 12A, 785-797.

Nahmias, S., Schmidt, C.P., 1984. An efficient heuristic for the multi-item newsboy problem with a single constraint. Naval Research Logistics 31, 463-474.
Niederhoff, J.A., 2007. Using separable programming to solve the multi-product multiple ex-ante constraint newsvendor problem and extensions. European Journal of Operational Research 176, 941-955.

Perry, M., Sohal, A.S., Rumpf, P., 1999. Quick Response supply chain alliances in the Australian textiles, clothing and footwear industry. International Journal of Production Economics 62, 119-132.

Press, W.H., Teukolsky, S.A., Vetterling, W.T., Flannery, B.P., 1992. Numerical Recipes in C: The Art of Scientific Computing, 2nd ed. Cambridge University Press, New York.

Raman, A., Kim, B., 2002. Quantifying the impact of inventory holding cost and reactive capacity on an apparel manufacturer's profitability. Production and Operations Management 11, 358-373.

Robert, C.P., 2007. The Bayesian Choice, 2nd ed. Springer, New York

Serel, D.A., 2009. Optimal ordering and pricing in a quick response system. International Journal of Production Economics 121, 700-714.

Sethi, S.P., Yan, H., Zhang, H., Zhou, J., 2007. A supply chain with a service requirement for each market signal. Production and Operations Management $16,322-342$.

Shao, Z., Ji, X., 2006. Fuzzy multi-product constraint newsboy problem. Applied Mathematics and Computation 180, 7-15.

Shi, J., Zhang, G., 2010. Multi-product budget-constrained acquisition and pricing with uncertain demand and supplier quantity discounts. International Journal of Production Economics 128, 322-331.

Silver, E.A., Pyke, D.F., Peterson, R., 1998. Inventory Management and Production Planning and Scheduling, 3rd ed. John Wiley \& Sons, New York.

Taleizadeh, A.A., Niaki, S.T.A., Hoseini, V., 2009. Optimizing the multi-product, multi-constraint, bi-objective newsboy problem with discount by a hybrid method of goal programming and genetic algorithm. Engineering Optimization 41, 437-457.

Topkis, D.M., 1979. Equilibrium points in non-zero sum $n$-person submodular games. SIAM Journal on Control and Optimization 17, 773-787.

Zhang, B., Du, S., 2010. Multi-product newsboy problem with limited capacity and outsourcing. European Journal of Operational Research 202, 107-113.

Zhang, B., Xu, X., Hua, Z., 2009. A binary solution method for the multi-product newsboy problem with budget constraint. International Journal of Production Economics 117, 136-141.

Zhang, G., 2010. The multi-product newsboy problem with supplier quantity discounts and a budget constraint. European Journal of Operational Research 206, 350-360.

Zhou, Y.J., Chen, X.H., Wang, Z.R., 2008. Optimal ordering quantities for multiproducts with stochastic demand: return-CVaR model. International Journal of Production Economics 112, 782-795.

Zhu, X., Mukhopadhyay, S.K., Yue, X., 2011. Role of forecast effort on supply chain profitability under various information sharing scenarios. International Journal of Production Economics 129, 284-291. 